Accepted for publication in the Astrophysical Journal

\title{
Spitzer mapping of molecular hydrogen pure rotational lines in NGC 1333: A detailed study of feedback in star formation
}

\author{
Sébastien Maret ${ }^{1}$, Edwin A. Bergin \\ Department of Astronomy, University of Michigan, 500 Church Street, Ann Arbor, MI \\ 48109-1042 \\ David A. Neufeld \\ Department of Physics and Astronomy, John Hopkins University, 3400 North Charles \\ Street, Baltimore, MD 21218 \\ Joel D. Green, Dan M. Watson \\ Department of Physics and Astronomy, University of Rochester, Rochester, NY 14627 \\ Martin O. Harwit \\ Department of Astronomy, Cornell University, Ithaca, NY 14853-6801; and 511 H Street \\ SW, Washington, DC 20024-2725 \\ Lars E. Kristensen \\ Leiden Observatory, P.O. Box 9513 NL-2300 RA Leiden, The Netherlands \\ Gary J. Melnick \\ Harvard-Smithsonian Center for Astrophysics, 60 Garden Street, Cambridge, MA 02138 \\ Paule Sonnentrucker \\ Department of Physics and Astronomy, John Hopkins University, 3400 North Charles \\ Street, Baltimore, MD 21218 \\ Volker Tolls
}

Harvard-Smithsonian Center for Astrophysics, 60 Garden Street, Cambridge, MA 02138

\footnotetext{
${ }^{1}$ Present address: Laboratoire d'Astrophysique de Grenoble, Observatoire de Grenoble, Université Joseph Fourier, CNRS, UMR 571, BP 53, F-38041 Grenoble, France
} 
Michael W. Werner, Karen Willacy

Jet Propulsion Laboratory, 4800 Oak Grove Drive, Pasadena, CA 91109

and

Yuan Yuan

Department of Physics and Astronomy, John Hopkins University, 3400 North Charles

Street, Baltimore, MD 21218

\begin{abstract}
We present mid-infrared spectral maps of the NGC 1333 star forming region, obtained with the the Infrared Spectrometer on board the Spitzer Space Telescope. Eight pure $\mathrm{H}_{2}$ rotational lines, from $S(0)$ to $S(7)$, are detected and mapped. The $\mathrm{H}_{2}$ emission appears to be associated with the warm gas shocked by the multiple outflows present in the region. A comparison between the observed intensities and the predictions of detailed shock models indicates that the emission arises in both slow $(12-24 \mathrm{~km} / \mathrm{s})$ and fast $(36-53 \mathrm{~km} / \mathrm{s})$ C-type shocks with an initial ortho-to-para ratio $\lesssim 1$. The present $\mathrm{H}_{2}$ ortho-to-para ratio exhibits a large degree of spatial variations. In the post-shocked gas, it is usually about 2 , i.e. close to the equilibrium value $(\sim 3)$. However, around at least two outflows, we observe a region with a much lower $(\sim 0.5)$ ortho-to-para ratio. This region probably corresponds to gas which has been heated-up recently by the passage of a shock front, but whose ortho-to-para has not reached equilibrium yet. This, together with the low initial ortho-to-para ratio needed to reproduce the observed emission, provide strong evidence that $\mathrm{H}_{2}$ is mostly in para form in cold molecular clouds. The $\mathrm{H}_{2}$ lines are found to contribute to $25-50 \%$ of the total outflow luminosity, and thus can be used to ascertain the importance of star formation feedback on the natal cloud. From these lines, we determine the outflow mass loss rate and, indirectly, the stellar infall rate, the outflow momentum and the kinetic energy injected into the cloud over the embedded phase. The latter is found to exceed the binding energy of individual cores, suggesting that outflows could be the main mechanism for core disruption.
\end{abstract}

Subject headings: astrochemistry — stars: formation — ISM: abundances ISM: molecules — ISM: individual () 


\section{Introduction}

The early phases of the formation of a star are characterized by the presence of bipolar outflows, which, as the material falls onto the young star, eject matter at high speeds (several tens of km/s) to distances up to tens of parsec away (e.g. Arce et al. 2007). These outflows have a great impact on the surrounding gas. They can clear out cavities in their natal molecular clouds, and may eventually cause cloud disruption. As they propagate through molecular clouds, they create shock waves that compress, heat up and alter the chemical composition of the gas. Theoretical studies of shock waves in the ISM (see Draine \& McKee 1993, for a review) predict that their nature depends on the shock velocity and the intensity of the magnetic field. Slow shocks ( $\leq 45 \mathrm{~km} / \mathrm{s}$ for typical values of the magnetic field) are of type $\mathrm{C}$ : the density, temperature and other quantities vary continuously through their passage. Furthermore, they are non-dissociative: molecules generally survive their passage, although the chemical composition of the gas can be significantly altered. On the other hand, faster shocks produce discontinuities in both the density and temperature of the gas, and may dissociate molecules on their passage.

Observations of $\mathrm{H}_{2}$ pure rotational lines in shock regions can provide important constraints on the physical and chemical conditions that prevail in these regions. For example, the $\mathrm{H}_{2}$ ortho-to-para ratio (hereinafter opr) is predicted to be greatly affected by the passage of a C-type shock. In the cold (pre-shock) gas, $\mathrm{H}_{2}$ is expected to be mostly in para form (Flower et al. 2006; Maret \& Bergin 2007). In the shocked gas, reactive collisions with $\mathrm{H}$ atoms can convert $\mathrm{p}-\mathrm{H}_{2}$ into o- $\mathrm{H}_{2}$ (Timmermann 1998; Wilgenbus et al. 2000). This reaction has a relatively high activation barrier $(\sim 4000 \mathrm{~K})$, and therefore the efficiency of the conversion depends critically on the temperature that is reached in the shock. Indeed, Wilgenbus et al. (2000) showed that effective para to ortho $\mathrm{H}_{2}$ conversion takes place in the temperature interval $700-1300 \mathrm{~K}$, while Kristensen et al. (2007) refined this result in to the interval $800-3200 \mathrm{~K}$. In addition, the efficiency of the conversion depends on the abundance of $\mathrm{H}$ atoms in the gas. Fast shocks produce higher $\mathrm{H}$ abundances, which makes the conversion faster.

Molecular hydrogen rotational lines have been observed in several star forming regions with the Infrared Space Observatory and, more recently, with the Spitzer Space Telescope (Werner et al. 2004). Using the Short Wavelength Spectrometer on board ISO, Neufeld et al. (1998) observed five pure rotational lines (from $0-0 S(1)$ to $0-0 S(5)$, hereafter simply referred as $S(1)$ and $S(5)$, respectively) towards the Herbig-Haro 54 outflow (HH 54). Using these multiple ortho and para lines, they determined simultaneously the rotational temperature and the ortho-to-para ratio. Interestingly, they measured an ortho-to-para ratio of 1.3 , which is significantly lower than the equilibrium value $(\sim 3)$ at the observed 
gas temperature $(\sim 650 \mathrm{~K})$. Lefloch et al. (2003) used the ISOCAM camera to map the $\mathrm{H}_{2} S(2)$ to $S(7)$ towards the $\mathrm{HH} 2$ outflow, and found important spatial variations in the opr (between 1.2 and 2.5) around the HH 2 object. Neufeld et al. (2006) used the Infrared Spectrometer (IRS, Houck et al. 2004) on board Spitzer to map the opr towards HH 54 and $\mathrm{HH}$ 7-11, and also observed a low, non-equilibrium opr, with important spatial variations. The fact that the opr is out-of-equilibrium suggests that the ortho-to-para conversion is relatively slow in these regions: the observed opr was interpreted as a fossil of an earlier epoch when the gas was cooler.

This paper presents complete $5.2-36.5 \mu \mathrm{m}$ spectral maps obtained with the IRS towards the NGC 1333 region in the Perseus cloud. The observations cover a region of roughly $6^{\prime} \times 10^{\prime}$, i.e. $0.4 \mathrm{pc} \times 0.5 \mathrm{pc}$ assuming a distance of $220 \mathrm{pc}($ Cernis 1990). To our knowledge, these are the largest complete spectral maps obtained with the IRS. NGC 1333 contains many YSOs, revealed by sub-millimeter (Sandell \& Knee 2001), millimeter (Lefloch et al. 1998) and midinfrared continuum maps (Gutermuth et al. 2008). It also contains numerous molecular outflows, detected for example in CO line emission (Knee \& Sandell 2000). Here we focus on $\mathrm{H}_{2}$ pure rotational line emission, from $S(0)$ to $S(7)$. These lines are used to map the $\mathrm{H}_{2}$ rotational temperature and opr over a large region, thus permitting detailed study of the impact of outflows on the surrounding cloud. The paper is organized as follow. In $\oiint_{2}$ we present the observations and discuss the data reduction. The spectra and maps that we obtained are presented in $\$ 3$. In $\$ 4$, we describe the analysis procedure that we used to derive the $\mathrm{H}_{2}$ column density, rotational temperature and opr from our observations. In $\$ 5$, we compare our observations with the predictions of shock models, we compare the variation of the opr with the temperature, and we discuss the impact of the outflows on the cloud. Our conclusions are presented in $\$ 6$.

\section{Observations}

NGC 1333 was observed using the IRS during Cycle 2 of the General Observer program, in March and September 2006. The Long-High (LH), Short-High (SH) and Short-Low (SL) modules of the IRS were used, providing a complete spectral coverage from 5.2 to $36.5 \mu \mathrm{m}$. The spectral resolution of these observations $(R=\lambda / \Delta \lambda)$ ranges from 64 to 128 for the SL mode, and is $\sim 600$ for the high resolution modules ( $\mathrm{SH}$ and $\mathrm{LH}$ ). The half-power beam size of the instrument ranges from $3^{\prime \prime}$ at $5.2 \mu \mathrm{m}$ to $10^{\prime \prime}$ at $38 \mu \mathrm{m}$ (Neufeld et al. 2006).

The SH observations consist of nine different Astronomical Observations Requests (AORs), each covering a rectangular region of $\sim 142^{\prime \prime} \times 120^{\prime \prime}$. Each of these AORs was obtained by moving the IRS slit in both perpendicular and parallel directions of the slit length, with a 
half-slit width stepping in perpendicular direction, and full-slit length in the parallel direction. Our SH observations do not cover the tip of the HH 7-11 outflow; therefore we have used archival data of this region obtained in February 2004 as part of the IRAC Guaranteed Time program, and that consists of two AORs of $58^{\prime \prime} \times 76^{\prime \prime}$ each. Details on these data can be found in Neufeld et al. (2006). When merged, the SH AORs cover a region of $\sim 6^{\prime} \times 10^{\prime}$, roughly centered on SVS $13\left(\alpha=17^{\mathrm{h}} 22^{\mathrm{m}} 38.2^{\mathrm{s}}\right.$ and $\left.\delta=-23^{\circ} 49^{\prime} 34.0^{\prime \prime} ; \mathrm{J} 2000\right)$. The LH observations consist of six AORs of $\sim 200^{\prime \prime} \times 273^{\prime \prime}$ each. As for the SH observations, these were obtained with a half-slit width stepping in perpendicular direction, and full-slit length in the parallel direction. These observations cover a region of $\sim 8^{\prime} \times 9^{\prime}$. Finally, the SL observations consist of six AORs of $\sim 202^{\prime \prime} \times 222^{\prime \prime}$, each obtained with a full-slit width stepping in the perpendicular direction, and half-slit width stepping in the parallel direction. These observations cover a region of $\sim 6^{\prime} \times 14^{\prime}$.

All the data, including the archival ones, have been reduced using the latest version (17.2) of the Spitzer Science Center pipeline. Further reduction was performed using the SMART software package (Higdon et al. 2004), supplemented by the IDL routines described in Neufeld et al. (2006). These routines remove the bad pixels in the LH and SH observations, extract spectra for each pixel along the slit of the different modules, spatially re-sample the spectra on a regular grid (with a spatial resolution corresponding to the pixel spacing, i.e. $2.3^{\prime \prime}, 1.8^{\prime \prime}$ and $4.5^{\prime \prime}$ for the SH, SL and LH modes, respectively), and finally produce continuum-subtracted spectral maps by fitting a Gaussian for each spectral line. A first look at our LH and SH spectral maps revealed the presence of stripes in the direction perpendicular to the slit length. As discussed in Neufeld et al. (2007), these stripes are due to the bad pixel extraction routine, which interpolates missing pixel values in the dispersion direction. Therefore, if a bad pixel is close to the central wavelength of a given line, the intensity measured at a given position along the slit is consistently underestimated, creating the observed stripes. Following Neufeld et al. (2007), we have corrected this effect by applying a correction factor for each line and each position along the slit (see Neufeld et al. 2007 for details on how this correction factor is determined). This technique was found to remove the stripes from our maps quite efficiently. Note that our LH observations were not affected by this problem because they were obtained with a half-slit length stepping in the parallel direction, as opposed to the SH and SL data which were obtained with a full-slit length stepping in that direction. Therefore, in LH mode, any given position on the sky was observed twice, and potential stripes were washed-out when averaging the spectra together. 


\section{Results}

\subsection{Spectra}

Fig. 11 to 3 present average spectra obtained on several positions of our maps. These spectra were obtained by averaging all the spectra observed within a 15" FWHM Gaussian aperture around a given position, in order to increase the signal-to-noise ratio. We detect eight pure rotational lines - from $S(0)$ to $S(7)$ - towards $\mathrm{HH}$ 7. On the other hand, no $\mathrm{H}_{2}$ emission is detected towards the NGC 1333-IRAS 4A and 4B Class 0 protostars. Several fine structure atomic lines are also detected, such as the Fe II ${ }^{4} F_{7 / 2}-{ }^{4} F_{9 / 2}(17.9 \mu \mathrm{m})$, the S I ${ }^{3} P_{1}-{ }^{3} P_{2}(25.2 \mu \mathrm{m})$, Fe II ${ }^{6} D_{7 / 2}-{ }^{6} D_{9 / 2}(26.0 \mu \mathrm{m})$ and Si II ${ }^{2} P_{3 / 2}^{0}-{ }^{2} P_{1 / 2}^{0}(34.8 \mu \mathrm{m})$ lines. Table 1 gives the intensities measured towards several positions for these lines. Thanks to an overlap in the wavelength range covered by the SL and SH modules, the $\mathrm{H}_{2} S(2)$ intensity was measured with each module, and was found to agree within $\sim 20 \%$.

Several PAH features - at 6.2, 7.2, 8.6, 11.2 and $12.8 \mu \mathrm{m}$ - as well as the $9.7 \mu \mathrm{m}$ silicates absorption band are clearly detected towards IRAS 8. Finally, the $\mathrm{CO}_{2}$ ice feature at $15.2 \mu \mathrm{m}$ is seen on IRAS 2. A discussion of all the detected spectral features is beyond the scope of this paper; in the following, we focus on the interpretation of the $\mathrm{H}_{2}$ line emission, and we postpone the discussion of other lines and spectral features to forthcoming papers.

\subsection{Maps}

Fig. 4 a-i show maps of the $\mathrm{H}_{2} S(0)$ to $S(7)$ lines. $\mathrm{H}_{2} S(1), S(2), S(3), S(4)$ and $S(5)$ emission is readily detected along several outflows. The $\mathrm{H}_{2} S(0), S(6)$ and $S(7)$ maps have a lower signal-to-noise ratio, and emission of these lines is barely detected at this spatial resolution (4.5 and $1.8^{\prime \prime}$ for the $\mathrm{LH}$ and SL modes, respectively). In the following, we discuss the morphology of the $\mathrm{H}_{2}$ emission along the different outflows.

\subsubsection{SVS 13 and $H H$ \%-11}

The Class I source SVS 13 (Strom et al. 1976) is associated with a chain of Herbig-Haro objects, HH 7-11. These objects are excited by a high-velocity molecular outflow, which is detected in $\mathrm{CO}$ and $\mathrm{SiO}$ rotational lines (Bachiller et al. 1998a, 2000, Knee \& Sandell 2000), as well as the $\mathrm{H}_{2} 1-0 S(1)$ ro-vibrational line at $2.12 \mu \mathrm{m}$ Aspin et al. 1994; Hodapp \& Ladd 1995; Khanzadyan et al. 2003). A second outflow, roughly orientated along a northsouth axis and driven by the SVS 13B Class 0 source, is detected in $\mathrm{SiO} J=2-1$ emission 
(Bachiller et al. 1998b).

The south-east lobe of the HH 7-11 outflow is clearly detected in our $S(1)$ map (Fig. 4 a). This map does not cover the north-west lobe, but emission from this lobe is seen in the $S(2)$ and $S(3)$ maps (Fig. $4 \mathrm{~b}$ and $4 \mathrm{c}$ ). $\mathrm{H}_{2} S(1)$ and $S(3)$ emission is also detected along the north-south outflow originating from SVS 13B. The position angle (from the north to east) of the $\mathrm{H}_{2}$ emission is about $160^{\circ}$. The emission extends as far as $\sim 3^{\prime}$ south of SVS 13B, up to the tip of the NGC 1333-IRAS 4A outflow (see below). Some weak emission along this flow is also seen in the $\mathrm{H}_{2} S(2)$ line. Diffuse $S(1)$ emission extends to the north along the same axis, and may be associated with this outflow as well (see 33.2 .5 ).

\subsection{2. $N G C$ 1333-IRAS 2}

NGC 1333-IRAS 2 (Jennings et al. 1987, hereafter IRAS 2) is a binary system composed of the IRAS $2 \mathrm{~A}$ and IRAS $2 \mathrm{~B}$ Class 0 protostars. This system is associated with two outflows that both originate $\sim 6^{\prime \prime}$ west of IRAS 2A (Knee \& Sandell 2000). The first one is roughly orientated along a north-south axis, and is detected in CO (Liseau et al. 1988; Sandell et al. 1994) and $\mathrm{H}_{2} 1-0 \mathrm{~S}(1)$ (Hodapp \& Ladd 1995). The second outflow is roughly oriented along an east-west axis (position angle $\sim 104^{\circ}$ ) and is detected in $\mathrm{CO}, \mathrm{CH}_{3} \mathrm{OH}$ and $\mathrm{SiO}$ (Sandell et al. 1994; Bachiller et al. 1998a; Knee \& Sandell 2000; Jørgensen et al. 2004).

In our maps, we observe a peak of $S(1), S(2)$ and $S(3)$ emission east of IRAS $2 \mathrm{~B}$ along the axis of the east-west outflow. The position of this peak corresponds to the peak of the CS and $\mathrm{CH}_{3} \mathrm{OH}$ emission, and presumably arises from a bow-shock (Langer et al. 1996; Bachiller et al. 1998a). No emission is seen west of the source, but our maps have limited coverage in that direction. Two $\mathrm{H}_{2}$ emission spots are also seen north and south of IRAS $2 \mathrm{~A}$. These two peaks lie at a position angle of $\sim 12^{\circ}$, consistent with the orientation of the $\mathrm{H}_{2} 1-0 S(1)$ jet, but slightly different from the position angle of the CO jet ( $\sim 25^{\circ}$ Sandell et al. 1994). No emission is seen towards the protostars.

\subsection{3. $N G C$ 1333-IRAS 4}

NGC 1333-IRAS 4 is also a binary system, composed of two Class 0 protostars, IRAS 4A and IRAS 4B. IRAS 4A drives a powerful and well collimated outflow that extends roughly over $4^{\prime}$, and is detected in $\mathrm{H}_{2} 1-0 S(1)$ (Hodapp \& Ladd 1995) as well as CO and SiO lines (Blake 1996; Knee \& Sandell 2000). A more compact outflow, with an inclination close to $90^{\circ}$ (i.e. almost perpendicular to the plane of the sky), is driven by IRAS 4B (Blake 1996). 
We detect $\mathrm{H}_{2} S(1)$ emission at the southwestern tip of the IRAS 4A outflow. Unfortunately, our SH maps do not cover other parts of the flow. However, $S(2)$ and $S(3)$ emission peaks are seen at to the north-east and south-west of IRAS $4 \mathrm{~A}$. $\mathrm{No}_{2}$ is detected from the IRAS 4B outflow, nor from IRAS 4A and IRAS 4B protostars themselves.

\subsection{4. $\quad N G C$ 1333-IRAS 7 and $H H 6$}

NGC 1333-IRAS 7 is associated with two sub-millimeter sources, SM 1 and SM 2 (Sandell \& Knee 2001). The former is coincident with the $\mathrm{cm}$ source VLA 27 (Rodríguez et al. 1999), as well as with a water maser (Henkel et al. 1986). A molecular outflow, oriented along a east-west axis and probably driven by SM 1, is detected in CO (Liseau et al. 1988; Knee \& Sandell 2000). This outflow is the driving source for the chain of HH objects located east of SM 1, which include $\mathrm{HH}$ 6. Our maps reveal bipolar $\mathrm{H}_{2}$ emission orientated along the same axis as the $\mathrm{CO}$ outflow.

\subsubsection{NGC 1333-IRAS 6 and HH 12}

NGC 1333-IRAS 6 is coincident with a PMS star (SVS 107) and the continuum cm source VLA 42 (Rodríguez et al. 1999). An elongated structure is also seen towards that source in the sub-mm maps of Sandell \& Knee (2001). west of NGC 1333-IRAS 6 is a chain of HH objects that include HH 12. Knee \& Sandell (2000) suggested that these objects are excited by the outflow originating from SVS 13B.

In our $\mathrm{H}_{2}$ maps we see some diffuse emission west of IRAS 6 that extends from the south down to the north west lobe of the SVS 13 outflow. Although this diffuse emission appears broadly aligned with the axis of the SVS 13B, it is difficult to say from the present data if the $\mathrm{H}_{2}$ emission arises in the shocked gas from this outflow.

\subsection{6. $N G C$ 1333-IRAS 8}

NGC 1333-IRAS 8 is part of the optical nebulosity north of the cloud (see Fig. 4 b). It is associated with a PMS star, SVS 3. Although the IRS spectrum is dominated by PAH features (see Fig. 1), we also detect extended $\mathrm{H}_{2} S(2), S(3)$ and $S(5)$ (but no $S(4)$ ) to the south east of the source. 


\section{Analysis}

\subsection{Rotational diagrams}

In Fig. 5, we show rotational diagrams obtained for 15" FWHM Gaussian apertures centered at several positions along the outflows: HH 7, IRAS 4A-SW, IRAS 2A-S, IRAS 7 and SVS 13B-S. These positions are indicated on the $\mathrm{H}_{2}$ maps (Fig. 4 a-i). Line intensities were corrected for extinction using the standard $\left(R_{v}=3.1\right)$ galactic dust opacity curve from Weingartner \& Draine (2001), assuming $A_{v}=2$ for all sources (the value measured towards HH 8; Gredel 1996). In each diagram and for each line, we have plotted $\ln \left(N_{u} /\left(g_{u} g_{s}\right)\right)$, where $N_{u}$ is the column density in the upper level, $g_{u}$ is the rotational degeneracy (equal to $2 J+1$ ) and $g_{s}$ is the spin degeneracy (3 for ortho, and 1 for para transitions). If the ortho-to-para ratio is equal to its high temperature limit (i.e. 3 ), and if $\mathrm{H}_{2}$ transitions are thermalized at a single temperature, then the observed values would line up on a straight line. Instead, the diagram show a "zig-zag" pattern, indicating that the ortho-to-para ratio is lower than 3 . In addition, the rotational diagrams show a positive curvature; the observed values cannot be fit with a single excitation temperature. This suggests that the observed $\mathrm{H}_{2}$ emission arises in a mixture of gas with different temperatures, as already observed in $\mathrm{HH} 7$ and $\mathrm{HH} 54$ by Neufeld et al. (2006). Following these authors, we have fitted the observations with two gas components (herein after the "warm" and the "hot" components). Note that this two temperature model is an approximation; the gas has more likely a continuous temperature distribution. The total $\mathrm{H}_{2}$ column density, ortho-to-para ratio and excitation temperature in each gas component were kept as free parameters, and were adjusted to fit the observations using a minimization routine. These three parameter can be constrained quite independently from each other. The ortho-to-para ratio is constrained from the "zig-zag" pattern in the rotational diagram. The rotational temperature is determined from the slope of the rotational diagram. Finally, the total $\mathrm{H}_{2}$ column density is determined from the ordinate of the points in the diagram. This procedure produced good fits to the data; in particular, no clear departures from the LTE is seen, even for the highest energy lines. The critical densities above which collisional excitation dominates over radiative de-excitation, and energy levels are populated according to Boltzmann's statistic - range between 5 and $4 \times 10^{5} \mathrm{~cm}^{-3}$ for the $S(0)$ and $S(7)$ lines respectively (assuming a kinetic temperature of $1000 \mathrm{~K}$; Le Bourlot et al. 1999). Therefore the highest energy lines $(S(6)$ and $S(7))$ must arise in relatively dense $\operatorname{gas}\left(>10^{5} \mathrm{~cm}^{-3}\right)$.

The best fit parameters for HH 7, IRAS 4A-SW, IRAS 2A-S, IRAS 7, SVS 13B-S are given in Table 2. The rotational temperature towards these sources is between $\sim 300$ and $\sim 600 \mathrm{~K}$ for the warm component, and $\sim 1000-1500 \mathrm{~K}$ for the hot component. We 
measure an opr of $0.3-0.7$ and $1.4-2.1$ in the warm and hot components, respectively 1 , For both components, the opr is much lower than its equilibrium value for the measured (rotational) gas temperatures; for kinetic temperatures higher than $300 \mathrm{~K}$, the equilibrium value is essentially 3. For all sources, we measure a higher opr in the hot component than in the warm component. Similar behavior was noted previously by Neufeld et al. (2006, 2007). The dependence of opr on temperature is discussed in further detail below. The $\mathrm{H}_{2}$ column densities are typically $\sim 2-6 \times 10^{19} \mathrm{~cm}^{2}$ and $\sim 3-6 \times 10^{18} \mathrm{~cm}^{2}$ in the warm and hot components, respectively. Thus, the column density of the hot component is more than an order of magnitude smaller than the column density of the warm component.

\section{2. $\mathrm{H}_{2}$ column density, rotational temperature and ortho-to-para ratio maps}

Thanks to their large spatial coverage, our observations essentially map the ortho-topara ratio, rotational temperature, and column density of $\mathrm{H}_{2}$ in the core of the NGC 1333 cloud. For this purpose, the $\mathrm{H}_{2}$ line maps were re-sampled on a common grid with a $5^{\prime \prime}$ spacing. Only regions of the sky that were observed with all three modules were considered. We then constructed a rotational diagram in each pixel of the resulting map. As mentioned above, the $\mathrm{H}_{2}$ rotational emission arises from at least two gas components. With three free parameters for each component (column density, opr and rotational temperature), we need at least six lines detected in a given pixel of the maps. In practice, few pixels in our maps meet this criterion. Therefore, when constructing the maps, only the $\mathrm{H}_{2} S(0)$ to $S(3)$ transitions were considered, which allows for greater spatial coverage of the region. Since the $S(0)$ to $S(3)$ line emission is dominated by the warm component (see Fig. 5), the column density, opr and rotational temperature derived using these lines correspond to that component.

On Fig. 6, 7 and 8 we provide maps of the $\mathrm{H}_{2}$ column density, rotational temperature and opr. These quantities could be measured along both lobes of the SVS 13 outflow and around IRAS 7. We could also map those at the south-west tip of the IRAS 4A outflow, as well at the northern, southern and western tips of the IRAS 2 outflows. Elsewhere in the map, we did not detect the $\mathrm{H}_{2} S(0), S(1), S(2)$ and $S(3)$ lines simultaneously, or the lines were detected with an insufficient signal-to-noise ratio. Where measured, some important variations in these quantities are seen. The $\mathrm{H}_{2}$ column density ranges from $3 \times 10^{18}$ to $10^{20}$ $\mathrm{cm}^{-2}$. The highest column densities are seen along the HH 7-11 outflow (see Fig. 6). The rotational temperature ranges between 200 and $650 \mathrm{~K}$, with a maximum reached close to the

\footnotetext{
${ }^{1}$ The best fit parameters we obtain for HH 7 differ slightly from those reported by Neufeld et al. (2006) on the same source. The reason is that we used new data in addition to those of Neufeld et al. (2006).
} 
position of $\mathrm{HH}$, as well as the IRAS $2 \mathrm{~N}$ position (see Fig. 7). Finally, the $\mathrm{H}_{2}$ ortho-to-para ratio ranges from 0.1 and 3 (Fig. 8).

Some important variations in both the rotational temperature and the opr are seen. Along the HH 7-11 outflow, between SVS 13 and HH 8, we measure a rotational temperature of $\sim 400 \mathrm{~K}$ and an opr ranging between 1.5 and 2. Around $\mathrm{HH} 7$, we measure a higher rotational temperature $(\sim 650 \mathrm{~K})$, but a lower opr $(\sim 0.5)$. The presence of hot gas with a low opr in front of the HH 7-11 has already been observed by Neufeld et al. (2006). They suggested that this gas has been compressed and heated-up by the passage of the shock, but the ortho-to-para ratio has not reached LTE yet. Along the northwestern lobe of this flow, the spectra are noisier, and large variations from one pixel to the next. Around IRAS 4SW, the rotational temperature and the opr were measured only in a few pixels making the comparison between these two quantities difficult. On the other hand, we observe some variation in the rotational temperature along the north and south lobes of the IRAS 2 outflow. Like in HH 7-11, the rotational temperature is higher at the tip of this outflow. However, no corresponding variation is seen in the opr. Some relatively hot gas $(\sim 500 \mathrm{~K})$ with a low opr $(\sim 1)$ is also observed around the eastern lobe of the IRAS 7 outflow. The variation of the opr as a function of the $T_{\text {rot }}$ are further discussed in Appendix A.

\section{Model and Discussion}

\subsection{Comparison of the observed emission with the predictions of a shock model}

In this section, we compare the observed $\mathrm{H}_{2}$ line emission towards several outflow positions with the predictions of a stationary, planar C-type shock model that includes ortho para interconversion (Flower \& Pineau des Forêts 2003). The shock model has several free parameters: the pre-shock density $n$, the pre-shock velocity $v$, the initial (pre-shock) orthoto-para ration $o p r_{i}$, and the intensity of the transverse magnetic field. The latter is expressed as $b=B_{t} n_{0}^{-1 / 2}$, where $B_{t}$ is the transverse magnetic field in $\mu \mathrm{G}$ and $n_{0}$ is the pre-shock density in $\mathrm{cm}^{-3}$. The model has been run for a grid of parameters (Kristensen et al. in prep), and the results of these computations are available on the web ${ }^{2}$.

In Fig. 9, we compare the column densities obtained from our observations on aperture averaged positions with those predicted by the shock model. As a first approach, we have tried to reproduce the observed column densities with a single component shock. The value

${ }^{2}$ http://www.strw.leidenuniv.nl/\$ \$sim\$kristensen/models 
of $b$ was set to 1 , and the other parameters of the model were adjusted until a good agreement with the observations was obtained. We found that the observed $S(0), S(1), S(2)$ emission were fairly well fitted for a pre-shock density of $10^{4} \mathrm{~cm}^{-3}$, an initial opr of 0.01 or 1, a pre-shock velocity of $12-24 \mathrm{~km} / \mathrm{s}$, and a beam filling factor $f f$ of typically $10 \%$. However, this model greatly underestimates the observed lines with higher energies. In other words, the temperature reached in a slow shock is not sufficient to explain the $S(3)$ to $S(7)$ line that we observe. This emission requires higher gas temperatures that are reached only in faster shocks. Therefore we have tried to match the observed column densities with a combination of slow and fast shock components. Both the pre-shock density and initial opr were kept the same as for the slow shock component, and the velocity of the fast shock component was adjusted in order to match the $S(3)$ to $S(7)$ lines. We found that the emission of these lines were well matched for a shock velocity of $36-53 \mathrm{~km} / \mathrm{s}$ and a filling factor of typically $1 \%$. In fact, this two component model provides an excellent fit to the observations (see Fig. 5), with the exception of the $S(0)$ line $\left[E_{\text {up }}=510 \mathrm{~K}\right]$ in IRAS-2, IRAS-7 and SVS13B-S, which is somewhat underestimated by the model. Possibly, this line arises in a separate, non-shock component towards these sources. Indeed, in their study of supernovae remnants, Neufeld et al. (2007) found that the $S(0)$ emission was more extended than other spectral lines they observed. In one source, they found evidence for both a shock excited $S(0)$ component and a diffuse emission component.

Table 3 gives the best-fit model parameters for the slow and fast shock components. Towards all positions, we obtain a good fit for a pre-shock density of $10^{4} \mathrm{~cm}^{-3}$. The shock velocity in the slow shock component is between 12 and $24 \mathrm{~km} / \mathrm{s}$, while that in the fast shock component is between 36 and $53 \mathrm{~km} / \mathrm{s}$. For all sources but IRAS 4-SW, the emission is well matched assuming an initial opr of 0.01. For the latter, we obtain a slightly better fit for an initial opr of 1.0. However, the grid of models covers only a relatively small sample of opr $_{i}(0.01,1,2$ and 3) so this parameter is not constrained precisely. It is likely that opr values ranging between 0.01 and 1 would also provide a good fit to the observations. The filling factor ranges from 2 to $15 \%$ for the slow component, and from 0.8 to $3 \%$ for the fast component. Since shocks driven by a collimated jet are not planar but have a bow-shape, it seems natural that we observe a range of velocities: at the apex of the bow, the shock is moving faster with respect to the wings of the bow (Smith et al. 1991). This high-velocity component has a small beam filling factor, because the high velocities are present only at the apex of the bow. On the other hand, slower gas - leading to lower excitation - is present in the broader wings of the bow.

It is worth noting that the fit is not be unique. For example, we found that the emission observed in HH 7 can also be fitted by a model with an higher pre-shock density $\left(10^{5}\right.$ $\mathrm{cm}^{-3}$ ), but a smaller pre-shock velocity $(15$ and $30 \mathrm{~km} / \mathrm{s}$ for the slow and fast component, 
respectively). In this case, the observations require an initial opr of 1 , because the para to ortho conversion at these shock velocities is not efficient enough. Another reasonable fit was found for a pre-shock density of $10^{6} \mathrm{~cm}^{-3}$ and pre-shock velocities of 10 and $20 \mathrm{~km} / \mathrm{s}$ for the slow and fast component, respectively. Therefore the pre-shock density and the pre-shock velocity are difficult to constrain simultaneously from the present observations. However, other constraints on these parameters have been obtained using FIR lines observations. Molinari et al. (2000) observed $\mathrm{CO}$ and $\mathrm{H}_{2}$ lines with ISO, and compared these observations with the predictions of Kaufman \& Neufeld (1996) shock models. They found that the observed emission was well reproduced by a shock with a velocity ranging between 15 and $20 \mathrm{~km} / \mathrm{s}$, and a pre-shock density of $10^{4} \mathrm{~cm}^{-3}$. Giannini et al. (2001) also compared the $\mathrm{H}_{2} \mathrm{O}$ and CO line emission observed with ISO-LWS towards the red-lobe of the IRAS 4 outflow to the model predictions of Kaufman \& Neufeld (1996). Their observations are consistent with a velocity ranging between 15 and $20 \mathrm{~km} / \mathrm{s}$, and a pre-shock density of $10^{4} \mathrm{~cm}^{-3}$ (see their Fig. 8). Thus the pre-shock density adopted here is consistent with the values derived by these authors using $\mathrm{CO}$ emission, which is a much more sensitive probe of density. It is also interesting to note that the shock velocity range they obtain agree well with what we obtain here for the slow shock component. Furthermore Smith et al. (2003) obtained images of the 2-1 S(1) and 1-0 S(1) $\mathrm{H}_{2}$ emission lines around the $\mathrm{HH} 7$ bow shock. They found that their observations were well reproduced by a C-type shock model with a pre-shock density of $8 \times 10^{3} \mathrm{~cm}^{-3}$, a bow speed of $55 \mathrm{~km} / \mathrm{s}$ and a magnetic field of $97 \mu \mathrm{G}$ (corresponding to $b \simeq 1$ for that pre-shock density). These parameters agree remarkably well with the values obtained here. The bow speed is slightly higher than the velocity we derive for the fast shock component $(45 \mathrm{~km} / \mathrm{s})$. This is consistent with models that predict that pure rotational $\mathrm{H}_{2}$ lines arise in the wings of the bow, where the shock velocity is smaller than at the apex (see Smith et al. 2003, Fig 5).

An important result of the present study is that the $\mathrm{H}_{2}$ observations cannot be reproduced by an initial ortho-to-para ratio greater than 1 ; models with opr $_{i}=2$ or 3 produce "flat" rotational diagrams, i.e. opr values that are close to the high temperature limit of 3. This indicates that $\mathrm{H}_{2}$ in the cold gas (i.e. before the passage of a shock) is mostly in para form. Flower et al. (2006) studied the evolution of the opr as a function of time in a pre-stellar core. In their computations, $\mathrm{H}_{2}$ is assumed to form on the grains with an opr of 3. Reactive collisions between $\mathrm{o}-\mathrm{H}_{2}$ and $\mathrm{p}-\mathrm{H}_{3}^{+}$(or o- $\mathrm{H}_{3}^{+}$) forms $\mathrm{p}-\mathrm{H}_{2}$, and consequently the opr decreases with time. The steady state value $\left(\sim 3 \times 10^{-3}\right.$ for $T=10 \mathrm{~K}$, and $\sim 3 \times 10^{-2}$ for $T=30 \mathrm{~K}$ ) is reached after $t \sim 3 \times 10^{7} \mathrm{yr}$, for a density of $10^{4} \mathrm{~cm}^{-3}$. Our observations and modelling suggest that the initial ortho-to-para ratio is lower than 1 . According to the Flower et al. (2006) computations, an ortho-to-para of 1 is reached after $3 \times 10^{6}$ year (see their Fig. 1); this is comparable to the life-time of nearby molecular clouds obtained by 
Hartmann et al. (2001). Therefore the value of the initial opr is consistent with that is expected from chemical models.

\subsection{Thermal history of the shocked gas}

The measure of the opr along the several outflows in the cloud provides constraints on the thermal history of the gas. Our observations and models suggest that in the cold gas, $\mathrm{H}_{2}$ is mostly in para form. Shock waves heat up the gas and trigger para-to-ortho conversion by reactive collisions with $\mathrm{H}$, so the opr increases. However, the reaction has a large activation barrier $(\sim 3900 \mathrm{~K})$, so it is only efficient for gas temperatures greater than a few thousand Kelvins. As the post-shock gas cools down, the interconversion becomes less efficient, and the opr remains frozen. Of course, reactions with $\mathrm{H}_{3}^{+}$will tend to decrease the opr in the post-shock gas. However, this occurs on relatively long timescales $\left(\sim 10^{6}\right.$ yr for $n_{\mathrm{H}}=10^{4}$; Flower et al. 2006), when compared to the dynamical timescale of the flows. Thus the opr retains a memory of the thermal history of the gas.

Constraints on the thermal history of the gas may be placed by simple considerations. If we assume that a parcel of the gas is warmed-up by a passage of a shock wave to a temperature $T$ for a duration $\tau$, and that ortho-para interconversion occurs only by reactive collisions with $\mathrm{H}$, then the fraction of $\mathrm{H}_{2}$ in ortho form in this parcel is given by (Neufeld et al. 2006):

$$
f_{\text {ortho }}(\tau)=f_{\text {ortho }}^{i} \exp \left(\frac{-n(\mathrm{H}) k \tau}{f_{\text {ortho }}^{\mathrm{LTE}}}\right)+f_{\text {ortho }}^{\mathrm{LTE}}\left[1-\exp \left(\frac{-n(\mathrm{H}) k \tau}{f_{\text {ortho }}^{\mathrm{LTE}}}\right)\right]
$$

where $f_{\text {ortho }}^{i}$ is the initial ortho fraction, $f_{\text {ortho }}^{\text {LTE }}$ is the ortho fraction at the local thermodynamic equilibrium, $n(\mathrm{H})$ is the hydrogen density, and $k=k_{p o}+k_{o p}$ is the sum of the rate coefficients for para-to-ortho and ortho-to-para conversion, given by $k=10^{-11} \exp (-3900 / T) \mathrm{cm}^{3} \mathrm{~s}^{-1}$ (Schofield 1967) ${ }^{3}$. Following Neufeld et al. (2006), we assume that $\mathrm{H}$ in the shocked gas is mainly produced by the formation of water from atomic oxygen, so that $n(\mathrm{H}) / n\left(\mathrm{H}_{2}\right) \sim 10^{-3}$. Thus, for a pre-shock $\mathrm{H}_{2}$ density of $10^{4} \mathrm{~cm}^{-3}, n(\mathrm{H}) \sim 10 \mathrm{~cm}^{-3}$. Fig. 10 shows the opr as a function of time for different gas temperatures, assuming an initial opr of 0.01 . In HH 7, we measure an opr of 0.37 and 1.99 in the warm $(T=611 \mathrm{~K})$ and hot $(T=1401 \mathrm{~K})$

\footnotetext{
${ }^{3}$ In the analogous equation given by (Neufeld et al. 2006), $k_{p o}$, the rate coefficient for para-to-ortho conversion alone, was erroneously given in place of $k$. The former is $25 \%$ smaller than the latter (a difference which is negligible relative to other uncertainties).
} 
components, respectively. This corresponds to timescales of $\sim 8 \times 10^{3}$ and $\sim \times 10^{3}$ yr for the warm and hot components, respectively.

The values we obtain can be compared to the prediction of shock models. The timescale during which the gas temperature is elevated on passing through a non dissociative shock is given by $N_{s} /\left(n_{0} v_{s}\right)$, where $N_{s}$ is the column density of the warm shocked gas, $n_{0}$ is the pre-shock density, and $v_{s}$ is the pre-shock velocity (Neufeld et al. 2006). Assuming a preshock density of $10^{4} \mathrm{~cm}^{-3}$, and a pre-shock velocity of $20 \mathrm{~km} \mathrm{~s}^{-1}$, we obtain, using the

analytical expression for $N_{s}$ from Neufeld et al. (2006), a shock timescale of $400 \mathrm{yr}$. This is about an order of magniture lower than the timescales we obtain for both the warm and hot components of HH 7. This may suggest that several shocks waves have passed through the quiescent gas and have progressively increased the opr, so $o p r_{i}$ is greater than 0.01. If we assume that $\mathrm{opr}_{i}$ is 0.37 - the value we observe in the warm component of $\mathrm{HH} 7$, at the tip of the HH 7-11 outflow - we found, using Eq. (1), that $800 \mathrm{yr}$ are needed to reach the value of 1.99 measured in the hot component, assuming a temperature of $1401 \mathrm{~K}$. This is broadly consistent with the 400 yr timescale obtain above.

\subsection{Global outflow properties}

We have obtained $5.2-35.8 \mu \mathrm{m}$ spectroscopic maps encompassing nearly the full spatial extent of 5 molecular outflows from embedded young protostars (HH 7-11, SVS 13B, IRAS 4A, IRAS 2, IRAS 7). In the following section we will explore how the information obtained in these maps provides a direct measurement of the mass loss rate and outflow energetics from these young stars.

\subsubsection{Determination of total outflow energy loss}

As shown in Fig. 4 a-i, we have detected optically thin emission from eight rotational transitions of $\mathrm{H}_{2}$ within these flows. Using these data, we can calculate the total $\mathrm{H}_{2}$ luminosity in rotational emission, $L_{\mathrm{H}_{2}}$, in the outflow by summing the emission over all rotational states and at each position within the region associated with each outflow. The transitions we have observed account for most of the luminosity carried by $\mathrm{H}_{2}$ rotational emissions, the brightest transitions falling within the range with $J_{u p}=2-9$ covered by Spitzer-IRS. We provide the value of $\mathrm{L}_{\mathrm{H}_{2}}$ for each flow in Table 4. The $\mathrm{H}_{2}$ luminosity is related to the total outflow cooling rate by $L_{\text {tot }}=\frac{1}{f_{c}} L_{\mathrm{H}_{2}}$, where $f_{c}$ is the fraction of the total cooling due to $\mathrm{H}_{2}$ rotational emissions. 
The shock models of Kaufman \& Neufeld (1996) can be used to explore the contribution of $\mathrm{H}_{2}$ to the overall cooling of the outflow via emission. Based on analysis of the $\mathrm{H}_{2}$ emission (§5.1) we find typical shock parameters of $n_{0} \sim 10^{4} \mathrm{~cm}^{-3}$ and $v_{s} \sim 10-50 \mathrm{~km} / \mathrm{s}$. Under these conditions Kaufman \& Neufeld (1996) find that $f_{c} \sim 0.2-0.7$. The majority of $\mathrm{H}_{2}$ cooling is via rotational lines, with only few percent contribution from $\mathrm{H}_{2}$ vibrational emission. The rest of the cooling flux arises from rotational, and to a lesser extent vibrational, lines of $\mathrm{H}_{2} \mathrm{O}$ and $\mathrm{CO}$, along with $[\mathrm{O} \mathrm{I}]$ fine structure emission.

The Infrared Space Observatory has observed the primary cooling lines of CO, [O I], $\mathrm{H}_{2} \mathrm{O}, \mathrm{OH}$, and $\mathrm{H}_{2}$ with the SWS and LWS spectrometers in some of our sources. Molinari et al. (2000) observed HH 7 and compile the luminosities of the major cooling lines within the bandpass. For the HH 7 shock they find that $4.8 \times 10^{-2} \mathrm{~L}_{\odot}$ is released by O I, $2 \times 10^{-2} \mathrm{~L}_{\odot}$ by $\mathrm{CO}, 0.7 \times 10^{-2} \mathrm{~L}_{\odot}$ by $\mathrm{H}_{2} \mathrm{O}$, and $2.3 \times 10^{-2} \mathrm{~L}_{\odot}$ by $\mathrm{H}_{2}$. However, the $\mathrm{O} \mathrm{I}, \mathrm{CO}$, and $\mathrm{H}_{2} \mathrm{O}$ observations were obtained using the LWS instrument with an $80^{\prime \prime}$ beam centered on $\mathrm{HH} 7$, while the $\mathrm{H}_{2}$ observations covered only the $S(1)-S(5)$ transitions within a $14^{\prime \prime} \times 20^{\prime \prime}$ SWS beam. Our observations in Fig. 4a-i demonstrate that the $\mathrm{H}_{2}$ emission extends well beyond the SWS aperture and the ISO SWS value is therefore a lower limit. We have convolved our data with a simulated $80^{\prime \prime}$ LWS beam and have recomputed the total luminosity of $\mathrm{H}_{2}$ from $S(0)-S(7)$. We find that the $\mathrm{H}_{2}$ luminosity in an $80^{\prime \prime}$ beam centered on $\mathrm{HH} 7$ is $0.1 \mathrm{~L}_{\odot}$. Note that in this source, the luminosity of the $\mathrm{H}_{2} 1-0 S(0)$ ro-vibration line is $3 \times 10^{-4} \mathrm{~L}_{\odot}$ (Khanzadyan et al. 2003), well below the $\mathrm{H}_{2}$ luminosity in rotational emission. Combining the ISO observations with this revised value, we find that the $\mathrm{H}_{2}$ provides about $50 \%$ of the total cooling $\left(f_{c} \sim 0.5\right)$, in good agreement with models.

Information for other flows can be gleaned from the summary of ISO cooling lines of Giannini et al. (2001) which included two of our sources: the IRAS 2 north-south flow, and the IRAS 4A outflow. For these sources, we find (after adapting the results of Giannini et al. to our adopted distance of $220 \mathrm{pc}$ ) that $f_{c} \sim 0.25$. Using this information the total cooling luminosity of the outflows in NGC 1333 is approximately $L_{t o t} \sim 2-4 L_{\mathrm{H}_{2}}$. For HH 7-11 we will adopt a $f_{c}=0.5$ and for all other flows (which are not as prominent as HH 7-11) we adopt $f_{c}=0.25$.

\subsubsection{Determination of the stellar mass loss by outflows}

The total energy loss generated by the outflow driven shock determined above is a direct measurement of the mechanical luminosity of the outflows. In this fashion, $\frac{1}{2} \dot{M}_{o} v_{s}^{2}=$ $\left(1-f_{m}\right) L_{t o t}=\left(1-f_{m}\right) \frac{1}{f_{c}} L_{\mathrm{H}_{2}}$, where $\left(1-f_{m}\right)$ is the fraction of shock mechanical energy that is translated into internal excitation, as opposed to translational motion. Kaufman \& 
Neufeld (1996) estimate that $\left(1-f_{m}\right) \sim 0.75$ and we can then use the $\mathrm{H}_{2}$ cooling emission to determine the outflow mass loss rate, $\dot{M}_{o}$ from the young star. This equation can be simplified and placed in terms of the observed $\mathrm{H}_{2}$ luminosity and estimated shock velocity:

$$
\dot{M}_{o} \simeq 10^{-6}\left(1-f_{m}\right) \frac{1}{f_{c}}\left(\frac{L_{\mathrm{H}_{2}}}{10^{-2} L_{\odot}}\right)\left(\frac{v_{s}}{10 \mathrm{~km} / \mathrm{s}}\right)^{-2} \mathrm{M}_{\odot} \mathrm{yr}^{-1} .
$$

To determine the shock velocity, we use the models of $\mathrm{H}_{2}$ emission discussed in $\S 5.1$. In general, the data is best fit by a combination of fast and slow shocks with the slow shock encompassing a larger filling factor within the beam. We have therefore estimated the average shock velocity for each flow by using a filling factor weighted average of the shock velocity based upon the model fits. In Table 4 we provide the observed $\mathrm{H}_{2}$ luminosity, assumed shock velocity, and derived outflow properties.

In general we find that the outflows from the Class 0 protostars in the NGC 1333 cloud have values $\dot{M}_{w} \sim 0.6-2 \times 10^{-6} \mathrm{M}_{\odot} \mathrm{yr}^{-1}$. These values are comparable to values estimated in the literature (e.g. Bontemps et al. 1996; Giannini et al. 2001; Hatchell et al. 2007). In some cases measurements include sources within our sample. For example, Giannini et al. (2001) use FIR cooling lines towards IRAS 4 to derive $\dot{M}_{w}=0.4-1.4 \times 10^{-6} \mathrm{M}_{\odot} \mathrm{yr}^{-1}$ where we estimate $\dot{M}_{w}=2 \times 10^{-6} \mathrm{M}_{\odot} \mathrm{yr}^{-1}$. The derived mass outflow rates can be related to the mass accretion rate $\left(\dot{M}_{a}\right)$ using theoretical models of outflow generation. While the details and results can vary, a typical number is $\dot{M}_{o} \simeq 0.1 \dot{M}_{a}$ (Pudritz et al. 2007), and our estimates provide $\dot{M}_{a} \sim 10^{-5} \mathrm{M}_{\odot} \mathrm{yr}^{-1}$. In this regard, di Francesco et al. (2001) independently derived a mass infall rate of $1 \times 10^{-4} \mathrm{M}_{\odot} \mathrm{yr}^{-1}$ towards IRAS 4A using P Cygni spectral line profiles of $\mathrm{H}_{2} \mathrm{CO}$. In addition, Maret et al. (2002) derived an accretion rate of $5 \times 10^{-5} \mathrm{M}_{\odot} \mathrm{yr}^{-1}$ by modelling the FIR water lines emission from IRAS $4 \mathrm{~A}$ and $4 \mathrm{~B}$ envelopes. This is in reasonable agreement with our estimate of $2 \times 10^{-5} \mathrm{M}_{\odot} \mathrm{yr}^{-1}$ for this source.

It is useful to place our results in a broader context. Previous estimates of these quantities for molecular outflows have generally used velocity-resolved $\mathrm{CO}$ emission to provide a measure of the total $\mathrm{H}_{2}$ mass in the flow, and used the velocity extent of the lines to trace the kinematics (e.g. Lada 1985; Bontemps et al. 1996). However, there are a number of uncertainties inherent in this method. The $\mathrm{CO}$ abundance has been calibrated to $\mathrm{H}_{2}$ in some clouds (e.g. Frerking et al. 1982), but can vary if some CO remains frozen on grains (e.g. Bergin \& Tafalla 2007) or is photodissociated by radiation generated in the shock or leaking through the outflow cavity. In addition, the $\mathrm{CO}$ molecules can be excited in both the low temperature natal core and in the warmer outflow. Thus towards the central core the spectral line contains a mixture of emission from the high velocity dispersion core and low dispersion core. To some extent these components can be disentangled, but there is 
significant uncertainty regarding the outflow mass present at low velocities that also correspond to the core (Lada \& Fich 1996). CO emission itself is often optically thick, even in the extended line wings. Observations of isotopologues are therefore required, but not always available to correct for the effects of optical depth (e.g. Yu et al. 1999; Arce \& Goodman 2001). Finally, estimates of the CO velocities are dependent on the outflow inclination. In a handful of cases, this can be directly measured if proper motion data can be used to measure the tangential velocity to compare to the observed radial velocity in the spectral lines. In the majority of cases this information is not available, and inclinations have instead been estimated by models of the flow velocity at different inclinations compared to the observed emission distribution (e.g. Cabrit et al. 1988). However, this estimate is also uncertain.

Our measurements do not suffer from any of these uncertainties. The $\mathrm{H}_{2}$ lines are optically thin and our observations encompass the most emissive $\mathrm{H}_{2}$ rotational lines, which we simply sum over rotational states and positions to derive the total emission. We have used shock models to calibrate the amount of cooling accounted for by other molecules. In 3 sources covered by our maps, we have confirmed that the models are accurately reproducing the observed distribution of cooling power. Since $\mathrm{H}_{2}$ is the dominant species and a primary coolant we are directly tracing the energy loss in the most abundant species. $\mathrm{H}_{2}$ does not emit within the core itself and thus we have no line of sight contamination. By using the cooling luminosity to trace outflow energetics our measurements are completely independent of the outflow inclination. As an example our momentum flux estimates are a factor of $\sim 4-8$ above values - uncorrected for inclination - derived from CO emission in same flows in NGC 1333 (Hatchell et al. 2007). On the other hand, our estimates are in good agreement the values (corrected for inclination) of Sandell \& Knee (2001).

Our conclusion is similar to that of Giannini et al. (2001) who used ISO observations of FIR cooling lines as a measure of the total mechanical luminosity. The major difference is that we use spatially resolved observations of $\mathrm{H}_{2}$ and supplement our results with those of Giannini et al. (2001) to derive the total cooling loss. Given that we have observed the major coolants, the primary uncertainty in the mass loss rate derivation is the assumed shock velocity. The expected range of shock strengths to create the observed $\mathrm{H}_{2}$ emission is $\sim 10-$ $50 \mathrm{~km} / \mathrm{s}$, with adopted values of $\sim 20-30 \mathrm{~km} / \mathrm{s}$ (Table 3). We therefore estimate that the mass outflow rate values are accurate to within a factor of 3 (given a distance of $220 \mathrm{pc}$ ).

\subsubsection{Impact of flow on natal core}

It is well known that outflows inject significant energy and momentum in the the surrounding cloud (e.g. Lada 1985; Arce et al. 2007; Davis et al. 2008) with some suggestions 
that these flows drive supersonic motions (Mac Low \& Klessen 2004). It is also possible that the outflow is the key player in the disruption of the natal core (e.g. Tafalla \& Myers 1997; Fuente et al. 2002). Our data offer a new opportunity to explore the question of the outflow impact on the surrounding material in the specific case of low mass star formation.

The total momentum injected by outflows into a core is given by $P=\dot{P} \tau_{d y n} \sim 0.3 \mathrm{M}_{\odot}$ $\mathrm{km} \mathrm{s}^{-1}$, where $\dot{P}=\dot{M}_{w} v_{s}$ and $\tau_{d y n}$ is the outflow dynamical timescale. Using $\tau_{d y n}$ values from Knee \& Sandell (2000), we find $P$ values ranging from 0.1 to $0.4 \mathrm{M}_{\odot} \mathrm{km} \mathrm{s}^{-1}$ (see Table 4 ). If a similar level of outflow activity persists during the lifetime of the embedded phase of $\sim 5 \times 10^{5}$ yrs (Evans et al. 2008) then the total momentum over this phase is $\sim 4-20$ $\mathrm{M}_{\odot} \mathrm{km} \mathrm{s}^{-1}$. There is some evidence in the literature of a decline in the outflow momentum flux during the transition from Class 0 to Class I (Bontemps et al. 1996) However, Hatchell \& Fuller (2008), with a homogeneous sample of sources in Perseus, do not confirm this result finding similar momentum fluxes for sources with comparable luminosities and masses.

Based on these estimates when the entrained outflowing material slows down to $1 \mathrm{~km}$ $\mathrm{s}^{-1}$ then it will have swept up $4-20 \mathrm{M}_{\odot}$ of material. Assuming a typical core mass of $\sim 5 \mathrm{M}_{\odot}$ then the outflow is clearly capable of destroying the core during the lifetime of the embedded phase. Of course, the flow will only disperse material in its path and the typical outflow cone angle in these sources is $\sim 60^{\circ}$ (Jørgensen et al. 2007). However, observations suggest that the outflow cone angle increases with age (Velusamy \& Langer 1998; Arce \& Sargent 2006). Estimates of core masses vary by nearly an order of magnitude in the literature (Lefloch et al. 1998; Sandell \& Knee 2001; Walsh et al. 2007) so definitive comparisons within our small sample are difficult. Nonetheless these data clearly suggest that the outflow could be the main mechanism for core dispersal.

To support our assertion that the transfer of outflow momentum to the core is a main mechanism for core dispersal we can also compare the energetics. Tafalla \& Myers (1997) demonstrated that the outflow from a massive star (Mon R2) exceeds or is comparable to the gravitational binding energy of its core. Thus the Mon R2 outflow has likely sculpted that object. However massive star outflows are more energetic than the low mass objects sampled in our maps and this result may not scale to low mass systems. The average total energy generated by the current generation of outflows in the center of NGC 1333 is $\left\langle L_{H_{2}} / f_{c} \times \tau_{\text {dyn }}\right\rangle=2 \times 10^{43}$ ergs. The gravitational binding energy of a sphere is $\alpha G M^{2} / R$ with $\alpha=1$ for cores with a $r^{-2}$ density profile (MacLaren et al. 1988), as expected for these embedded protostars. For a typical core mass of $\sim 5 \mathrm{M}_{\odot}$ and radius of $0.03 \mathrm{pc}$ the binding energy is $\sim 4 \times 10^{43} \mathrm{ergs}$. If we assume that a similar level of activity exists over the lifetime of the embedded phase then the outflow energetics are equal to or even exceed the gravitational energy of the core. In sum, both the flow energetics and momentum suggest 
that outflows are the primary method for core dispersal. Molecular gas observations show that NGC 1333 cores discussed here are embedded in a much larger but lower density cloud (e.g. Pineda et al. 2008). As typical for GMCs, the cloud binding energy is much greater than those of each individual cores. Thus the flows which disrupt the cores are not major players in cloud disruption as the cloud momentum and energy far exceed that provided by the current generation of flows over their lifetime.

\section{Conclusions}

We have presented Spitzer-IRS maps of seven pure rotational $\mathrm{H}_{2}$ lines in the NGC 1333 star forming region. These observations cover a region roughly $20^{\prime} \times 13^{\prime}$ that encompass a number of YSOs and outflows. We have analysed these observations using the rotational diagram technique in order to derive the excitation temperature and the $\mathrm{H}_{2}$ ortho-to-para ratio. Furthermore, these observations have been compared with the predictions of a planar stationary shock model. Finally, we have used the $\mathrm{H}_{2}$ total luminosity to estimate the mass outflow rates, the mass infall rate onto the central objects, and the momentum and energy injected into the cores and the cloud. Our main conclusions are as follow.

- $\mathrm{H}_{2}$ line emission is detected along several outflows in NGC 1333. In particular, we detect line emission along both lobes of the HH 7-11 outflow, along the south-west lobe of the outflow driven by IRAS 4A, along the north, south and east lobes of the IRAS 2 outflow, as well as around IRAS 7. In addition, we detect some faint emission south of SVS 13, which is associated with the outflow originating from SVS 13B. Diffuse emission is detected north of this source, and might be associated with this outflow as well.

- A rotational diagram analysis on several aperture averaged spectra indicate the presence of at least two gas components towards each position. The warm component has a rotational temperature between $\sim 300$ and $600 \mathrm{~K}$ and an opr between $\sim 0.3$ and 0.7 , while the hot component has a rotational temperature between $\sim 1000$ and 1500 $\mathrm{K}$ and an opr between $\sim 1.9$ and 2.2 .

- Comparison of the line fluxes measured towards these positions with the predictions of a planar stationary shock model indicate the presence of slow and fast shock components. A good fit of the observation is obtained for an initial opr of 0.01 (except in one source where the data are better fit with an initial opr of 1), a pre-shock density of $10^{4}$, and a shock velocity of $\sim 20 \mathrm{~km} / \mathrm{s}$ and $\sim 45 \mathrm{~km} / \mathrm{s}$ the slow and fast shock components, respectively. This indicates, in agreement with earlier studies, that $\mathrm{H}_{2}$ is mostly in para 
form in dense molecular clouds (i.e. opr $\lesssim 1$ ). This is consistent with the predictions of chemical models, provided that the cloud lifetime exeed a few million years.

- Maps of the rotational temperature and the opr in warm components show some important variations of these quantities across the mapped region. We confirm the presence of gas with a relatively high rotational temperature and low opr at the tip of the HH 7-11 outflow. This gas is probably "fresh gas" with a low initial opr that has been heated-up by the passage of a shock, but whose opr hasn't had time to increase significantly. The same feature is observed on both lobes of the IRAS 7 flow, although less clearly. Spatial averages of the opr and the rotational temperature along the flow support this hypothesis. Interestingly, no spatial correlation between the two quantities is found.

- Comparison between the total $\mathrm{H}_{2}$ luminosity and the luminosity of other lines observed by ISO in HH 7-11 and IRAS 4 indicates that between a fourth and half of the total cooling in these outflows occurs through the $\mathrm{H}_{2}$ lines. Therefore $\mathrm{H}_{2}$ lines can be used to estimate the kinetic energy injected into the natal cloud by these flows, as well as outflow mass loss rate which are found to be $0.6-2 \times 10^{-6} \mathrm{M}_{\odot} \mathrm{yr}^{-1}$. The latter quantity also places indirect constraints on the mass accretion rate onto the protostar: dynamical models predict that the outflow mass loss rate is typically 10 times lower than the accretion rate. Using the dynamical timescale obtained from millimeter observations, we derived the total momentum injected in the clouds by the outflows, and compared it to the core and cloud binding energy. We found that outflows have the potential to disrupt individual cores, but are probably not major players in cloud disruption.

This work is based on observations made with the Spitzer Space Telescope, which is operated by the Jet Propulsion Laboratory, California Institute of Technology under a contract with NASA. Support for this work was provided by NASA through an award issued by JPL/Caltech for program \#20378.

Facilities: Spitzer (IRS)

\section{A. Variation of the opr with the temperature}

As mentioned in $\S 4.2$, some important spatial variations in both the opr and the rotational temperature are observed in NGC 1333. In particular, we observe, at a tip of 
the HH 7-11 outflow, a bow shock-like region with a low opr $(\sim 0.5)$ and high rotational temperature ( $\sim 650 \mathrm{~K}$; see Fig. 11). This low opr region wraps nicely around the $\mathrm{H}_{2} S(1)$ rotational line emission, and suggests that it corresponds to gas which has been recently heated-up by the passage of a shock, but whose opr has not had time to reach the equilibrium value. Interestingly, this region also corresponds to the part of the flow where the rotational temperature is the highest. Around IRAS 7, we also observe low opr regions around the $\mathrm{H}_{2}$ $S(1)$ emission contours (see Fig. 12), but variations from one pixel to the other are important and it is less clear if this region correspond to high temperature gas, like in HH 7-11.

It is interesting to compare the variations of the opr and the rotational temperature as a function of the position of the flow in a more quantitative fashion. In order to do this, we have computed the average of the opr and rotational temperature along the outflows that show some degree of symmetry, and where both quantities were measured in a sufficient number of pixels. In order to "capture" the spatial variations, we have defined a series of ellipses of the same eccentricity, oriented along the flow and with an apex that is centered on the outflow driving source. The eccentricity of the ellipses has been chosen in order to match the shape of each outflow lobe as observed in the $\mathrm{H}_{2} S(1)$ emission. We have then computed the average of the opr ratio and the rotational temperature in the region between two consecutive ellipses (that is two ellipses with different major axes). On Fig. 11 and 12 . we show how those ellipses where defined for HH 7-11, IRAS 7 (east and west lobes) and IRAS 2 (south lobe). We have not computed the average for the other outflow lobes, because the opr ratio and the rotational temperature had greater uncertainties.

Fig. 13 shows the average opr and rotational temperature, as a function of the ellipse semi-major axis. Along the HH 7-11 outflow, we see a sharp drop in the average opr ratio $35^{\prime \prime}$ from SVS 13. Interestingly, the temperature does not seem to be correlated with the opr. It increases slightly 0 and $35^{\prime \prime}$ (although at a $1 \sigma$ level), and then starts to decrease. The highest temperature is reached where the opr ratio is minimal. Presumably, the hottest point corresponds to the shock front. In the post-shock gas, the para to ortho conversion has increased the opr, but the conversion is incomplete and the ratio does not reach the LTE value. In the pre-shock gas the opr has also probably increased with respect to its initial value, but has not had time to reach the same value that we see in the post-shock gas (i.e. $\sim 2$ ). Note that for a pre-shock density of $10^{4} \mathrm{~cm}^{-3}$, and a shock velocity of 20 $\mathrm{km} / \mathrm{s}$, the model predicts a shock front width - defined as the region of the shock where the temperature is greater than 1000 - of $\sim 10^{3}$ AU, i.e. about $5^{\prime \prime}$ at the distance of NGC 1333 .

The interpretation of the opr and rotational temperature along the other flows is less straightforward. We see an increase of the rotational temperature along the IRAS 4 lobe, but the maximum temperature is reached at the tip of the outflow; farther away no $\mathrm{H}_{2}$ emission 
is detected and the rotational temperature and the opr could not be determined. Unlike the $\mathrm{HH} 7-11$, the average opr ratio is roughly constant, and is $\sim 2$. The temperature in the east lobe of IRAS 7 flow seems to slightly decrease (note that the first ellipse contains only a couple of points, so the average is not very meaningful in this bin), while the opr is almost constant. On the other hand, the opr ratio and temperature may vary in a similar fashion that in HH 7-11. The opr drops sharply between 5 and $15^{\prime \prime}$, while the temperature seems to slightly increase in the same region. However, further away the temperature appears to be roughly constant.

\section{REFERENCES}

Arce, H. G. \& Goodman, A. A. 2001, ApJ, 554, 132

Arce, H. G. \& Sargent, A. I. 2006, ApJ, 646, 1070

Arce, H. G., Shepherd, D., Gueth, F., Lee, C.-F., Bachiller, R., Rosen, A., \& Beuther, H. 2007, in Protostars and Planets V, ed. B. Reipurth, D. Jewitt, \& K. Keil, 245-260

Aspin, C., Sandell, G., \& Russell, A. P. G. 1994, A\&AS, 106, 165

Bachiller, R., Codella, C., Colomer, F., Liechti, S., \& Walmsley, C. M. 1998a, A\&A, 335, 266

Bachiller, R., Gueth, F., Guilloteau, S., Tafalla, M., \& Dutrey, A. 2000, A\&A, 362, L33

Bachiller, R., Guilloteau, S., Gueth, F., Tafalla, M., Dutrey, A., Codella, C., \& Castets, A. 1998b, A\&A, 339, L49

Bergin, E. A. \& Tafalla, M. 2007, ARA\&A, 45, 339

Blake, G. A. 1996, in IAU Symp. 178: Molecules in Astrophysics: Probes \& Processes, 31-+

Bontemps, S., Andre, P., Terebey, S., \& Cabrit, S. 1996, A\&A, 311, 858

Cabrit, S., Goldsmith, P. F., \& Snell, R. L. 1988, ApJ, 334, 196

Cernis, K. 1990, Ap\&SS, 166, 315

Davis, C. J., Scholz, P., Lucas, P., Smith, M. D., \& Adamson, A. 2008, MNRAS, 387, 954

di Francesco, J., Myers, P. C., Wilner, D. J., Ohashi, N., \& Mardones, D. 2001, ApJ, 562, 770 
Draine, B. T. \& McKee, C. F. 1993, ARA\&A, 31, 373

Evans, II, N. J., Dunham, M. M., Jørgensen, J. K., Enoch, M. L., Merín, B., van Dishoeck, E. F., Alcalá, J. M., Myers, P. C., Stapelfeldt, K. R., Huard, T. L., Allen, L. E., Harvey, P. M., van Kempen, T., Blake, G. A., Koerner, D. W., Mundy, L. G., Padgett, D. L., \& Sargent, A. I. 2008, ArXiv e-prints

Flower, D. R. \& Pineau des Forêts, G. 2003, MNRAS, 343, 390

Flower, D. R., Pineau Des Forêts, G., \& Walmsley, C. M. 2006, A\&A, 449, 621

Frerking, M. A., Langer, W. D., \& Wilson, R. W. 1982, ApJ, 262, 590

Fuente, A., Martın-Pintado, J., Bachiller, R., Rodrıguez-Franco, A., \& Palla, F. 2002, A\&A, 387,977

Giannini, T., Nisini, B., \& Lorenzetti, D. 2001, ApJ, 555, 40

Gredel, R. 1996, A\&A, 305, 582

Gutermuth, R. A., Myers, P. C., Megeath, S. T., Allen, L. E., Pipher, J. L., Muzerolle, J., Porras, A., Winston, E., \& Fazio, G. 2008, ApJ, 674, 336

Hartmann, L., Ballesteros-Paredes, J., \& Bergin, E. A. 2001, ApJ, 562, 852

Hatchell, J. \& Fuller, G. A. 2008, A\&A, 482, 855

Hatchell, J., Fuller, G. A., \& Richer, J. S. 2007, A\&A, 472, 187

Henkel, C., Guesten, R., \& Haschick, A. D. 1986, A\&A, 165, 197

Higdon, S. J. U., Devost, D., Higdon, J. L., Brandl, B. R., Houck, J. R., Hall, P., Barry, D., Charmandaris, V., Smith, J. D. T., Sloan, G. C., \& Green, J. 2004, PASP, 116, 975

Hodapp, K.-W. \& Ladd, E. F. 1995, ApJ, 453, 715

Houck, J. R., Roellig, T. L., van Cleve, J., Forrest, W. J., Herter, T., Lawrence, C. R., Matthews, K., Reitsema, H. J., Soifer, B. T., Watson, D. M., Weedman, D., Huisjen, M., Troeltzsch, J., Barry, D. J., Bernard-Salas, J., Blacken, C. E., Brandl, B. R., Charmandaris, V., Devost, D., Gull, G. E., Hall, P., Henderson, C. P., Higdon, S. J. U., Pirger, B. E., Schoenwald, J., Sloan, G. C., Uchida, K. I., Appleton, P. N., Armus, L., Burgdorf, M. J., Fajardo-Acosta, S. B., Grillmair, C. J., Ingalls, J. G., Morris, P. W., \& Teplitz, H. I. 2004, ApJS, 154, 18 
Jennings, R. E., Cameron, D. H. M., Cudlip, W., \& Hirst, C. J. 1987, MNRAS, 226, 461

Jørgensen, J. K., Bourke, T. L., Myers, P. C., Di Francesco, J., van Dishoeck, E. F., Lee, C.-F., Ohashi, N., Schöier, F. L., Takakuwa, S., Wilner, D. J., \& Zhang, Q. 2007, ApJ, 659, 479

Jørgensen, J. K., Hogerheijde, M. R., Blake, G. A., van Dishoeck, E. F., Mundy, L. G., \& Schöier, F. L. 2004, A\&A, 415, 1021

Kaufman, M. J. \& Neufeld, D. A. 1996, ApJ, 456, 611

Khanzadyan, T., Smith, M. D., Davis, C. J., Gredel, R., Stanke, T., \& Chrysostomou, A. 2003, MNRAS, 338, 57

Knee, L. B. G. \& Sandell, G. 2000, A\&A, 361, 671

Kristensen, L. E., Ravkilde, T. L., Field, D., Lemaire, J. L., \& Pineau Des Forêts, G. 2007, A\&A, 469, 561

Lada, C. J. 1985, ARA\&A, 23, 267

Lada, C. J. \& Fich, M. 1996, ApJ, 459, 638

Langer, W. D., Castets, A., \& Lefloch, B. 1996, ApJ, 471, L111

Le Bourlot, J., Pineau des Forêts, G., \& Flower, D. R. 1999, MNRAS, 305, 802

Lefloch, B., Castets, A., Cernicharo, J., Langer, W. D., \& Zylka, R. 1998, A\&A, 334, 269

Lefloch, B., Cernicharo, J., Cabrit, S., Noriega-Crespo, A., Moro-Martín, A., \& Cesarsky, D. 2003, ApJ, 590, L41

Liseau, R., Sandell, G., \& Knee, L. B. G. 1988, A\&A, 192, 153

Mac Low, M.-M. \& Klessen, R. S. 2004, Reviews of Modern Physics, 76, 125

MacLaren, I., Richardson, K. M., \& Wolfendale, A. W. 1988, ApJ, 333, 821

Maret, S. \& Bergin, E. A. 2007, ApJ, 664, 956

Maret, S., Ceccarelli, C., Caux, E., Tielens, A. G. G. M., \& Castets, A. 2002, A\&A, 395, 573

Molinari, S., Noriega-Crespo, A., Ceccarelli, C., Nisini, B., Giannini, T., Lorenzetti, D., Caux, E., Liseau, R. ., Saraceno, P., \& White, G. J. 2000, ApJ, 538, 698 
Neufeld, D. A., Hollenbach, D. J., Kaufman, M. J., Snell, R. L., Melnick, G. J., Bergin, E. A., \& Sonnentrucker, P. 2007, ApJ, 664, 890

Neufeld, D. A., Melnick, G. J., \& Harwit, M. 1998, ApJ, 506, L75

Neufeld, D. A., Melnick, G. J., Sonnentrucker, P., Bergin, E. A., Green, J. D., Kim, K. H., Watson, D. M., Forrest, W. J., \& Pipher, J. L. 2006, ApJ, 649, 816

Pineda, J. E., Caselli, P., \& Goodman, A. A. 2008, ApJ, 679, 481

Pudritz, R. E., Ouyed, R., Fendt, C., \& Brandenburg, A. 2007, in Protostars and Planets V, ed. B. Reipurth, D. Jewitt, \& K. Keil, 277-294

Rodríguez, L. F., Anglada, G., \& Curiel, S. 1999, ApJS, 125, 427

Sandell, G. . \& Knee, L. B. G. 2001, ApJ, 546, L49

Sandell, G., Knee, L. B. G., Aspin, C., Robson, I. E., \& Russell, A. P. G. 1994, A\&A, 285, L1

Schofield, K. 1967, Planet. Space Sci., 15, 643

Smith, M. D., Brand, P. W. J. L., \& Moorhouse, A. 1991, MNRAS, 248, 451

Smith, M. D., Khanzadyan, T., \& Davis, C. J. 2003, MNRAS, 339, 524

Strom, S. E., Vrba, F. J., \& Strom, K. M. 1976, AJ, 314

Tafalla, M. \& Myers, P. C. 1997, ApJ, 491, 653

Timmermann, R. 1998, ApJ, 498, 246

Velusamy, T. \& Langer, W. D. 1998, Nature, 392, 685

Walawender, J., Bally, J., \& Reipurth, B. 2005, AJ, 129, 2308

Walsh, A. J., Myers, P. C., Di Francesco, J., Mohanty, S., Bourke, T. L., Gutermuth, R., \& Wilner, D. 2007, ApJ, 655, 958

Weingartner, J. C. \& Draine, B. T. 2001, ApJ, 548, 296

Werner, M. W., Roellig, T. L., Low, F. J., Rieke, G. H., Rieke, M., Hoffmann, W. F., Young, E., Houck, J. R., Brandl, B., Fazio, G. G., Hora, J. L., Gehrz, R. D., Helou, G., Soifer, B. T., Stauffer, J., Keene, J., Eisenhardt, P., Gallagher, D., Gautier, T. N., Irace, W., Lawrence, C. R., Simmons, L., Van Cleve, J. E., Jura, M., Wright, E. L., \& Cruikshank, D. P. 2004, ApJS, 154, 1 
Wilgenbus, D., Cabrit, S., Pineau des Forêts, G., \& Flower, D. R. 2000, A\&A, 356, 1010

Yu, K. C., Billawala, Y., \& Bally, J. 1999, AJ, 118, 2940 


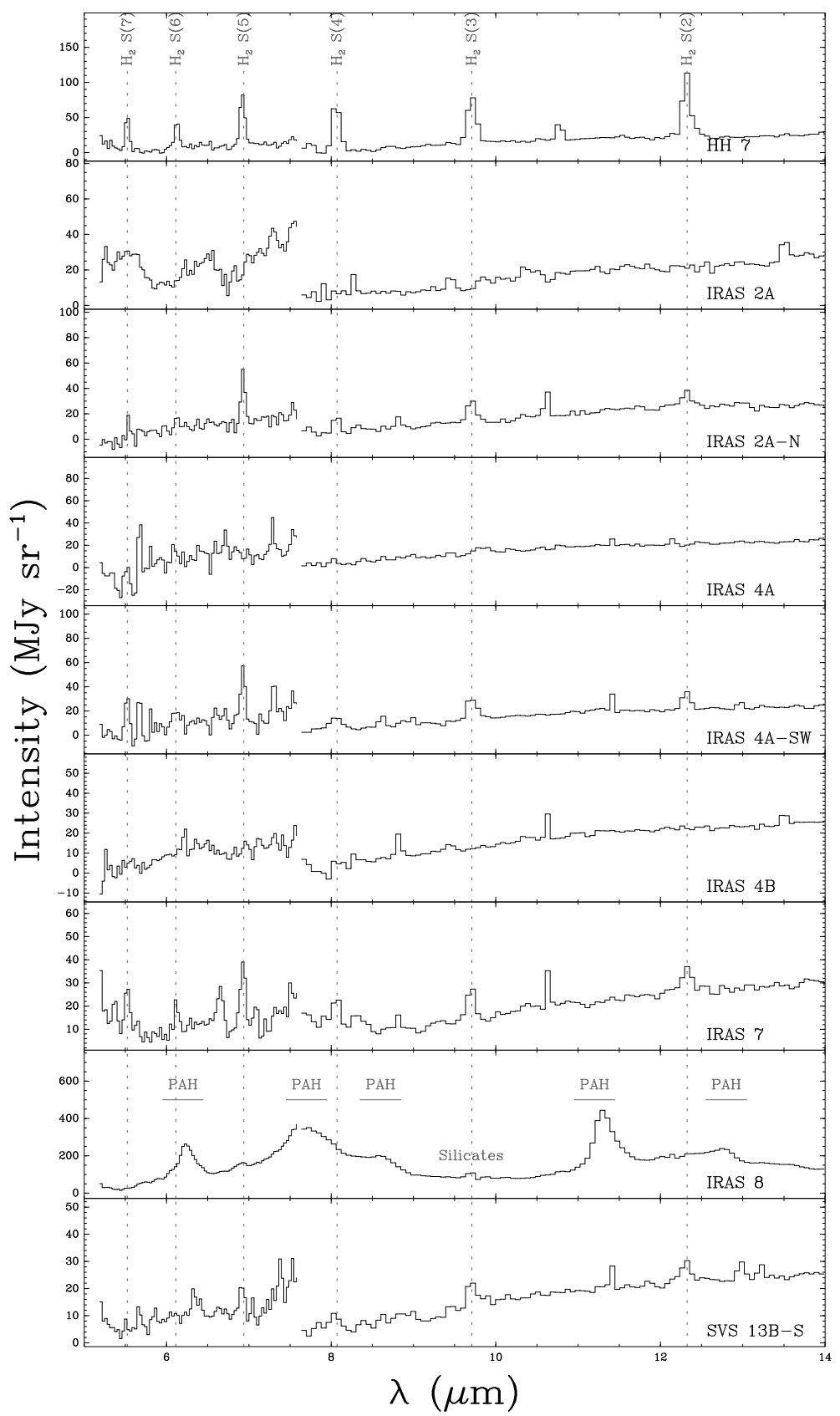

Fig. 1.- Averaged spectra observed with the SL module for $15^{\prime \prime}$ Gaussian apertures centered, from top to bottom, on HH $7\left(\alpha=03^{\mathrm{h}} 29^{\mathrm{m}} 08.45^{\mathrm{s}}, \delta=+31^{\circ} 15^{\prime} 29.2^{\prime \prime}\right.$; J2000) IRAS $2 \mathrm{~A}\left(\alpha=03^{\mathrm{h}} 28^{\mathrm{m}} 55.59^{\mathrm{s}}, \delta=+31^{\circ} 14^{\prime} 37.3^{\prime \prime} ; J 2000\right)$, IRAS $2 \mathrm{~A}-\mathrm{S}\left(\alpha=03^{\mathrm{h}} 28^{\mathrm{m}} 54.04^{\mathrm{s}}\right.$, $\left.\delta=+31^{\circ} 13^{\prime} 31.2^{\prime \prime} ; \mathrm{J} 2000\right)$, IRAS $4 \mathrm{~A}\left(\alpha=03^{\mathrm{h}} 29^{\mathrm{m}} 10.29^{\mathrm{s}}, \delta=+31^{\circ} 13^{\prime} 31.8^{\prime \prime} ; \mathrm{J} 2000\right)$, IRAS 4A-SW $\left(\alpha=03^{\mathrm{h}} 29^{\mathrm{m}} 06.62^{\mathrm{s}}, \delta=+31^{\circ} 12^{\prime} 17.7^{\prime \prime} ; \mathrm{J} 2000\right)$, IRAS $4 \mathrm{~B}\left(\alpha=03^{\mathrm{h}} 29^{\mathrm{m}} 11.99^{\mathrm{s}}\right.$, $\left.\delta=+31^{\circ} 13^{\prime} 08.9^{\prime \prime} ; \mathrm{J} 2000\right)$, IRAS $7\left(\alpha=03^{\mathrm{h}} 29^{\mathrm{m}} 11.31^{\mathrm{s}}, \delta=+31^{\circ} 18^{\prime} 31.1^{\prime \prime} ; \mathrm{J} 2000\right)$, IRAS $8\left(\alpha=03^{\mathrm{h}} 29^{\mathrm{m}} 12.5^{\mathrm{s}}, \delta=+31^{\circ} 22^{\prime} 08^{\prime \prime} ; \mathrm{J} 2000\right)$, and SVS 13B-S $\left(\alpha=03^{\mathrm{h}} 29^{\mathrm{m}} 05.0^{\mathrm{s}}\right.$, $\left.\delta=+31^{\circ} 13^{\prime} 14^{\prime \prime} ; \mathrm{J} 2000\right)$. Pure rotational $\mathrm{H}_{2}$ transitions are indicated, as well as the $6.2,7.2$, 8.6, 11.2 and $12.8 \mu \mathrm{m}$ PAH features, and the $9.7 \mu \mathrm{m}$ silicates absorption band. The spectra between $\sim 5$ and $7.5 \mu \mathrm{m}$ and between $\sim 7.5$ and $14 \mu \mathrm{m}$ correspond to the second and first order of the module, respectively. 


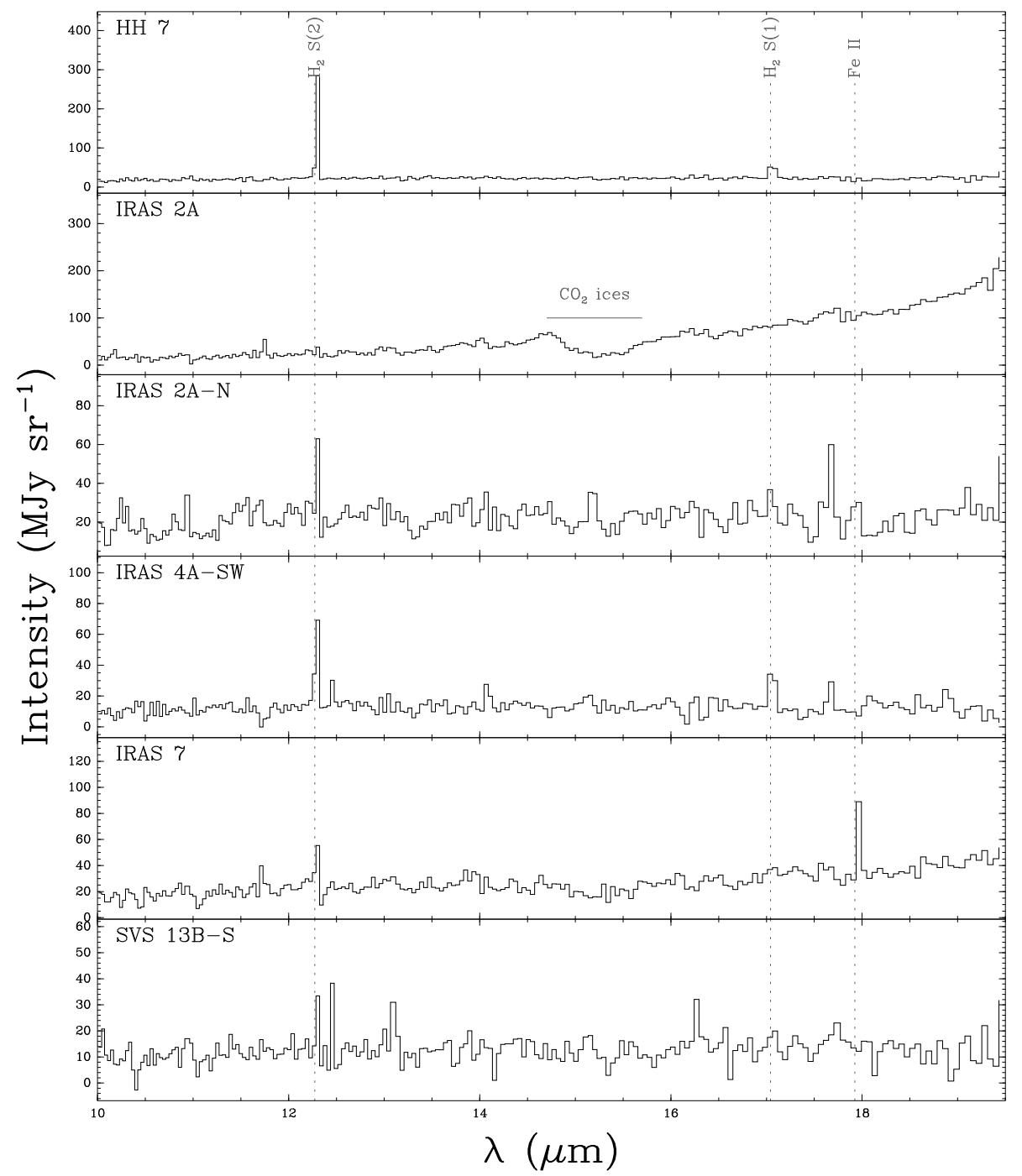

Fig. 2.- Same as Fig. 1 for the $\mathrm{SH}$ module. The position of the $15.2 \mu \mathrm{CO}_{2}$ ice feature is indicated. For clarity the spectral resolution has been degraded by a factor 4 . 


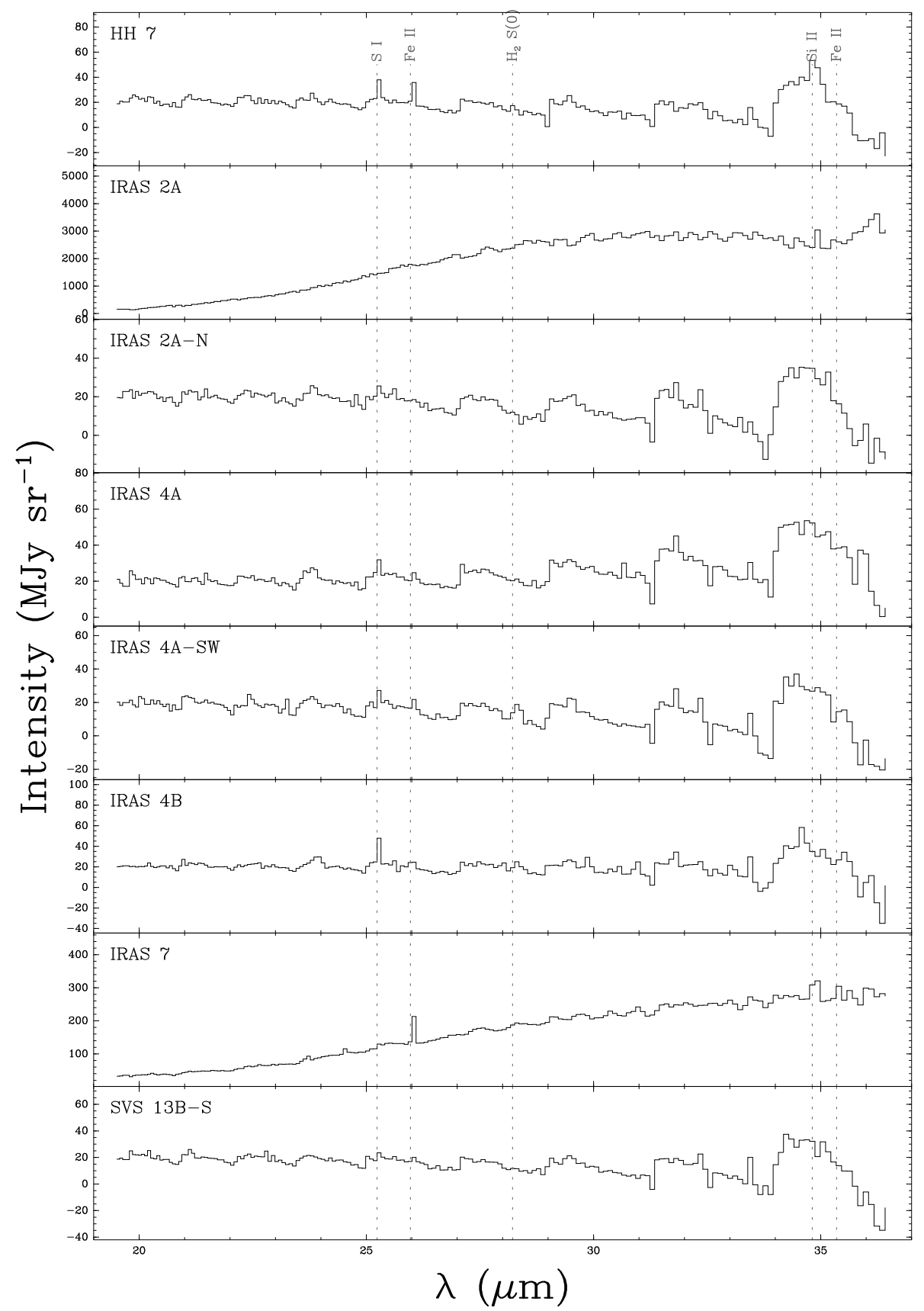

Fig. 3.- Same as Fig. 1 for the LH module. For clarity the spectral resolution has been degraded by a factor 4 . The steps seen in some of these spectra are caused by offsets in response of the different orders of the module. 


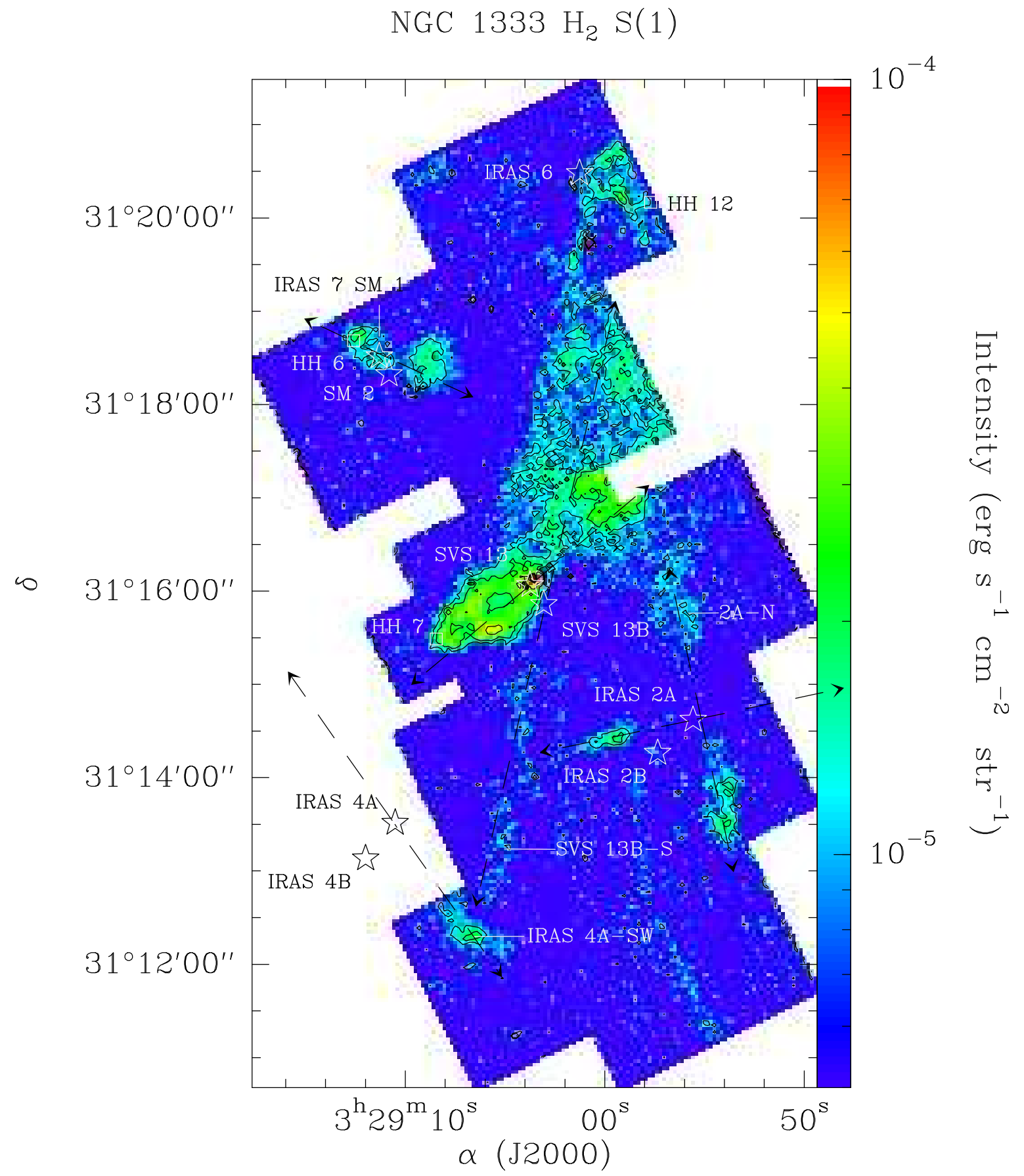

Fig. 4 a. $-\mathrm{H}_{2} S(1)$ continuum subtracted map obtained with the $\mathrm{SH}$ module. YSOs are indicated by open stars, while several $\mathrm{HH}$ objects are indicated by open squares. The outflows discussed in the text are indicated by dashed arrows. The two positions along the IRAS 2 and IRAS 4A outflows that are discussed in the text, IRAS 2A-N and IRAS 4A-SW, are also shown. Contours show the 3,5 and $10 \sigma$ noise levels. See the electronic edition of the Journal for panels $d-i$. 


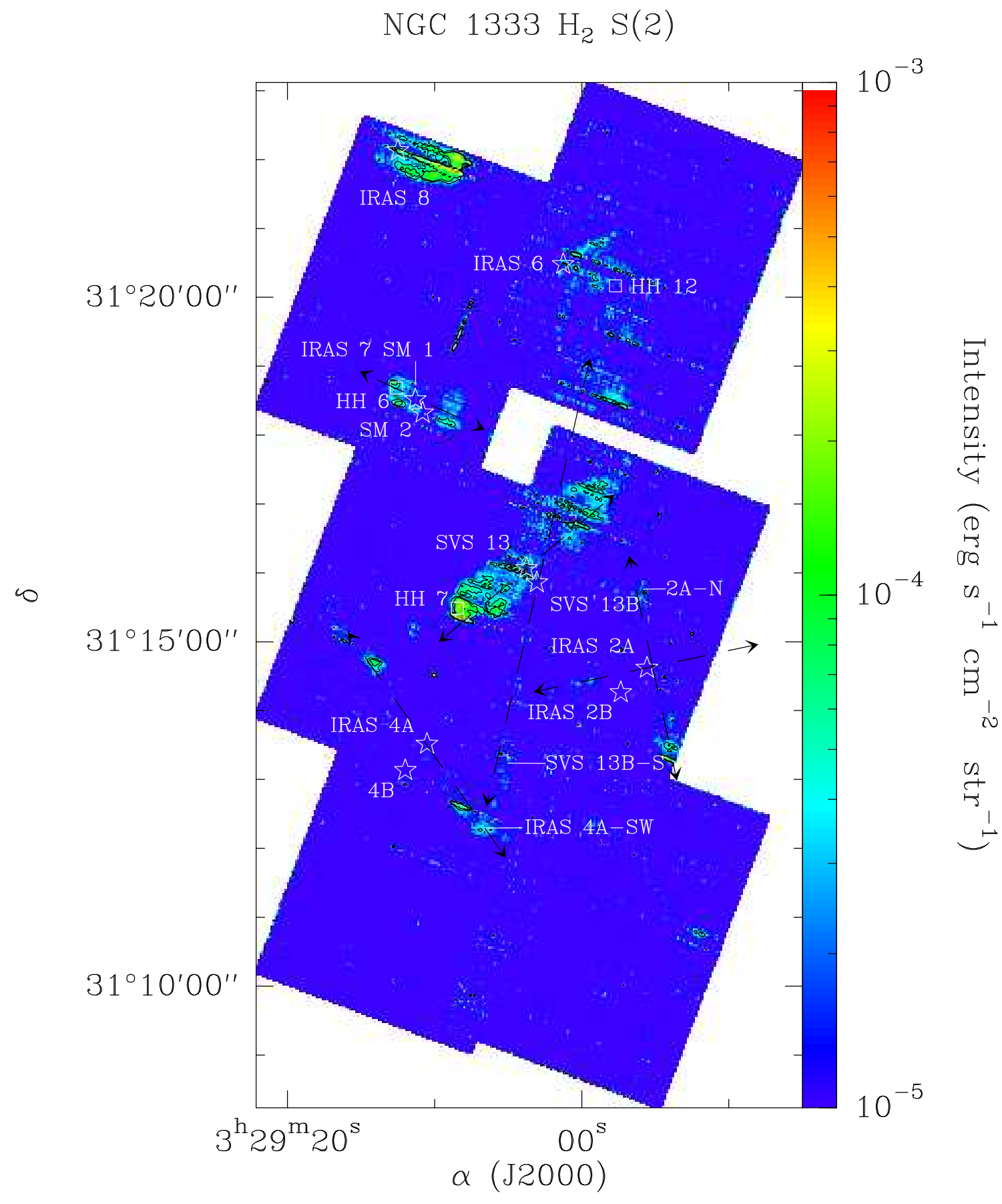

Fig. 4 b. $-\mathrm{H}_{2} S(2)$ map obtained with the SL module. Contours show the 3 and $5 \sigma$ noise level. 


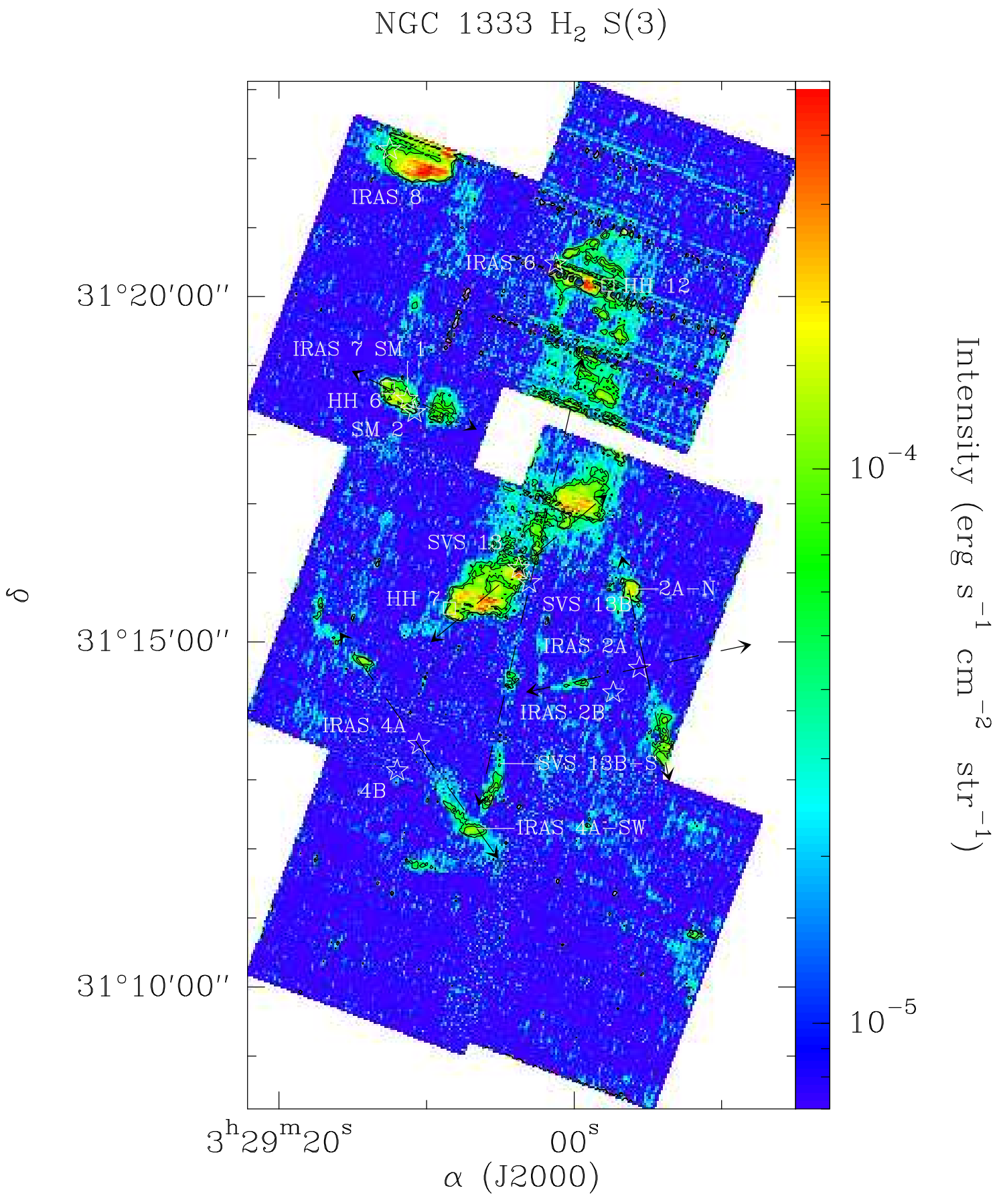

Fig. 4 c. $-\mathrm{H}_{2} S(3)$ map obtained with the SL module. Contours show the 3 and $5 \sigma$ noise level. 


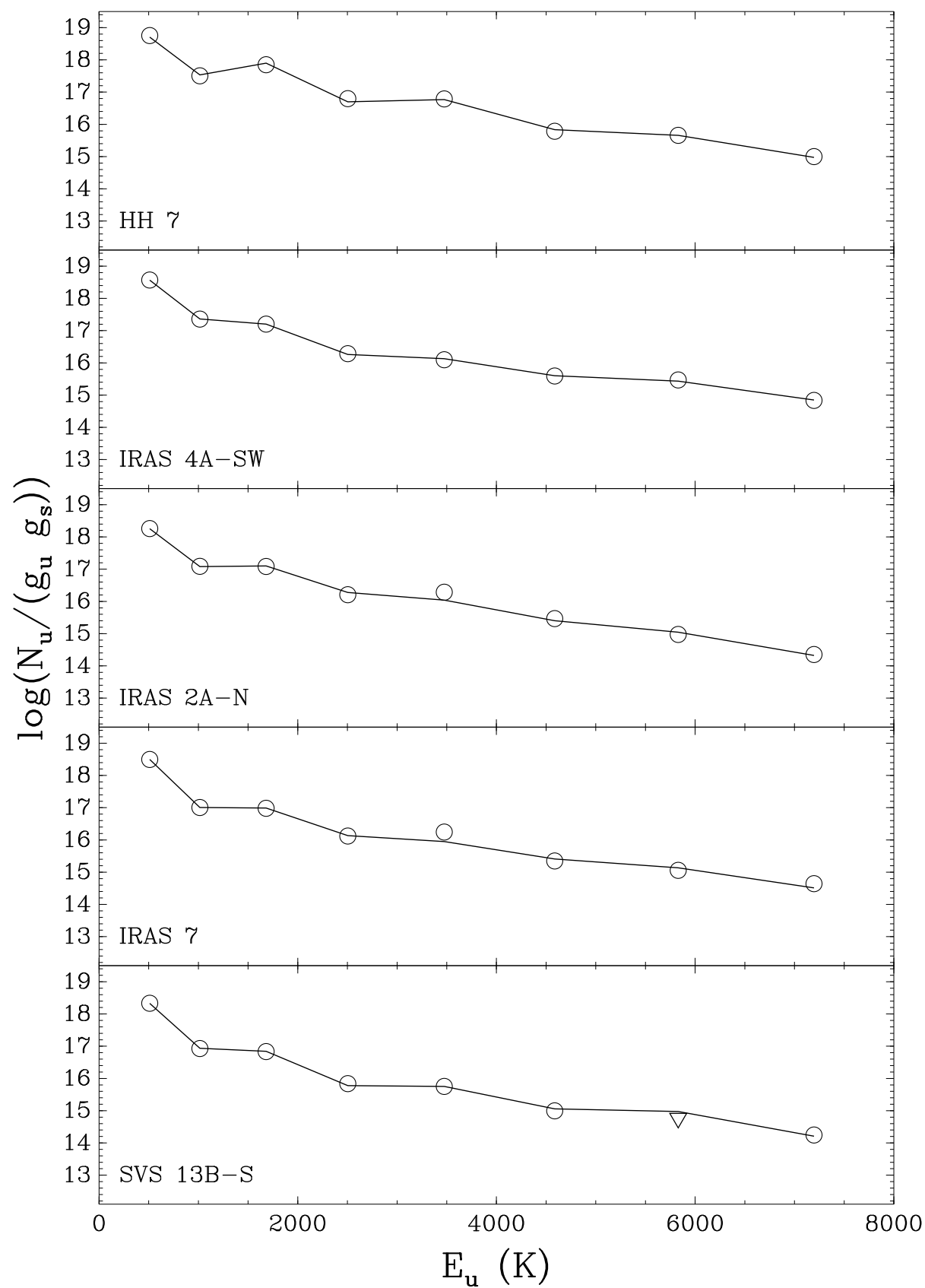

Fig. 5.- $\mathrm{H}_{2}$ rotational diagrams obtained for $15^{\prime \prime}$ Gaussian apertures centered on $\mathrm{HH} 7$, IRAS 4A-SW, IRAS 2A-S, IRAS 7 and SVS 13B-S. The black open circles correspond to the observations. The black open triangle is a $1 \sigma$ upper limit. The black solid lines are rotational diagram obtained assuming that two gas components, a warm and a hot one, are present (see $\S 4.1$ ). 


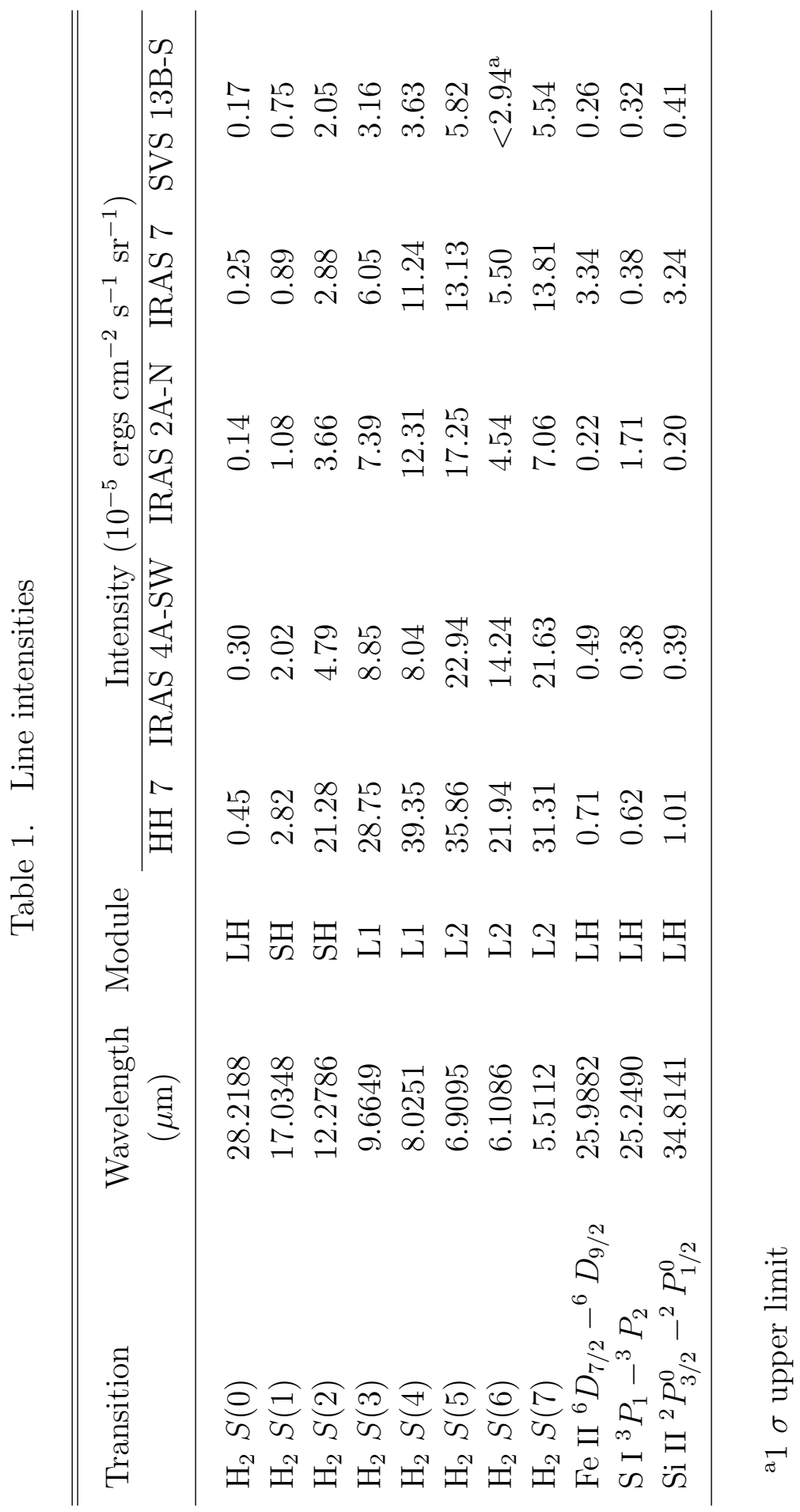


Table 2. Rotational diagrams results

\begin{tabular}{|c|c|c|c|c|c|c|}
\hline \multirow[b]{2}{*}{ Source } & \multicolumn{3}{|c|}{ Warm component } & \multicolumn{3}{|c|}{ Hot component } \\
\hline & $\begin{array}{l}N\left(\mathrm{H}_{2}\right) \\
\left(\mathrm{cm}^{-2}\right)\end{array}$ & $\begin{array}{l}T_{\text {rot }} \\
(\mathrm{K})\end{array}$ & opr & $\begin{array}{l}N\left(\mathrm{H}_{2}\right) \\
\left(\mathrm{cm}^{-2}\right)\end{array}$ & $\begin{array}{l}T_{\text {rot }} \\
(\mathrm{K})\end{array}$ & opr \\
\hline HH 7 & $5.9 \times 10^{19}$ & 611 & 0.37 & $6.2 \times 10^{18}$ & 1401 & 1.99 \\
\hline IRAS 4A-SW & $5.9 \times 10^{19}$ & 342 & 0.69 & $3.4 \times 10^{18}$ & 1513 & 1.94 \\
\hline IRAS 2A-N & $2.0 \times 10^{19}$ & 371 & 0.33 & $5.6 \times 10^{18}$ & 1055 & 2.09 \\
\hline IRAS 7 & $4.2 \times 10^{18}$ & 300 & 0.31 & $3.3 \times 10^{18}$ & 1268 & 2.11 \\
\hline SVS 13B-S & $3.2 \times 10^{19}$ & 312 & 0.51 & $1.5 \times 10^{18}$ & 1337 & 1.43 \\
\hline
\end{tabular}

Table 3. Best fit shock model parameters for aperture-averaged positions

\begin{tabular}{|c|c|c|c|c|c|c|c|c|}
\hline \multirow[b]{2}{*}{ Source } & \multicolumn{4}{|c|}{ Slow shock component } & \multicolumn{4}{|c|}{ Fast shock component } \\
\hline & $\begin{array}{c}n(\mathrm{H}) \\
\left(\mathrm{cm}^{-3}\right)\end{array}$ & $\begin{array}{c}v \\
\left(\mathrm{~km} \mathrm{~s}^{-1}\right)\end{array}$ & $o p r_{i}$ & $\begin{array}{l}f f \\
(\%)\end{array}$ & $\begin{array}{c}n(\mathrm{H}) \\
\left(\mathrm{cm}^{-3}\right)\end{array}$ & $\begin{array}{c}v \\
\left(\mathrm{~km} \mathrm{~s}^{-1}\right)\end{array}$ & $o p r_{i}$ & $\begin{array}{l}f f \\
(\%)\end{array}$ \\
\hline HН 7 & $10^{4}$ & 22 & 0.01 & 15 & $10^{4}$ & 45 & 0.01 & 3 \\
\hline IRAS 4A-SW & $10^{4}$ & 12 & 1 & 12 & $10^{4}$ & 41 & 1 & 2 \\
\hline IRAS $2 \mathrm{~A}-\mathrm{S}$ & $10^{4}$ & 23 & 0.01 & 4 & $10^{4}$ & 50 & 0.01 & 1.5 \\
\hline IRAS 7 & $10^{4}$ & 24 & 0.01 & 2.2 & $10^{4}$ & 53 & 0.01 & 1.1 \\
\hline SVS 13B-S & $10^{4}$ & 14 & 0.01 & 3 & $10^{4}$ & 36 & 0.01 & 0.8 \\
\hline
\end{tabular}




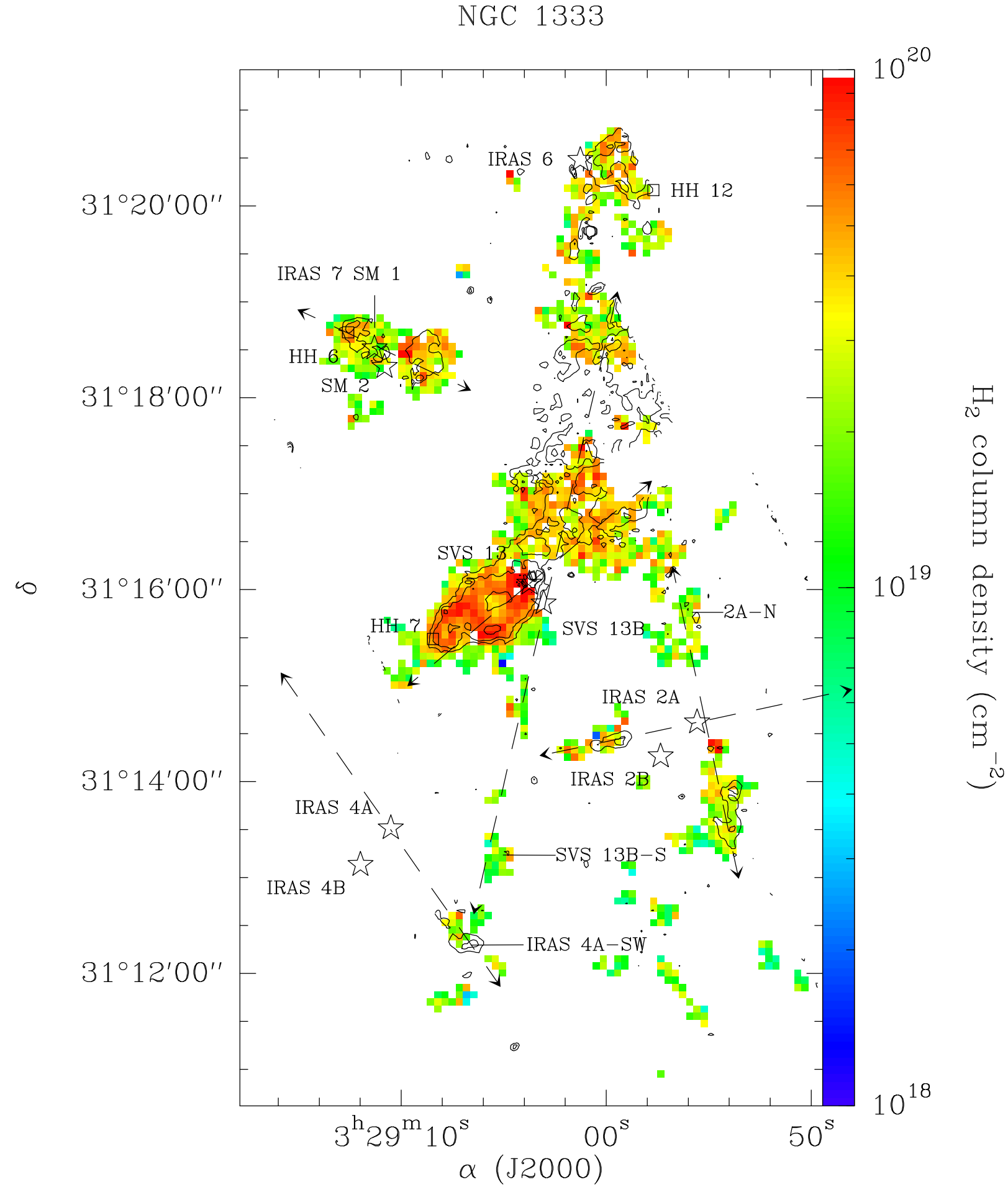

Fig. 6.- $\mathrm{H}_{2}$ column density map (color points) on top of the $\mathrm{H}_{2} S(1)$ emission (black contours). Regions of the map that appear in white correspond to points where a rotational diagram could not be constructed because of missing data or insufficient signal-to-noise ratio. Contour levels are those of the $S(1)$ emission taken from Fig. 4 a. Pixel size is $5^{\prime \prime}$. 
NGC 1333

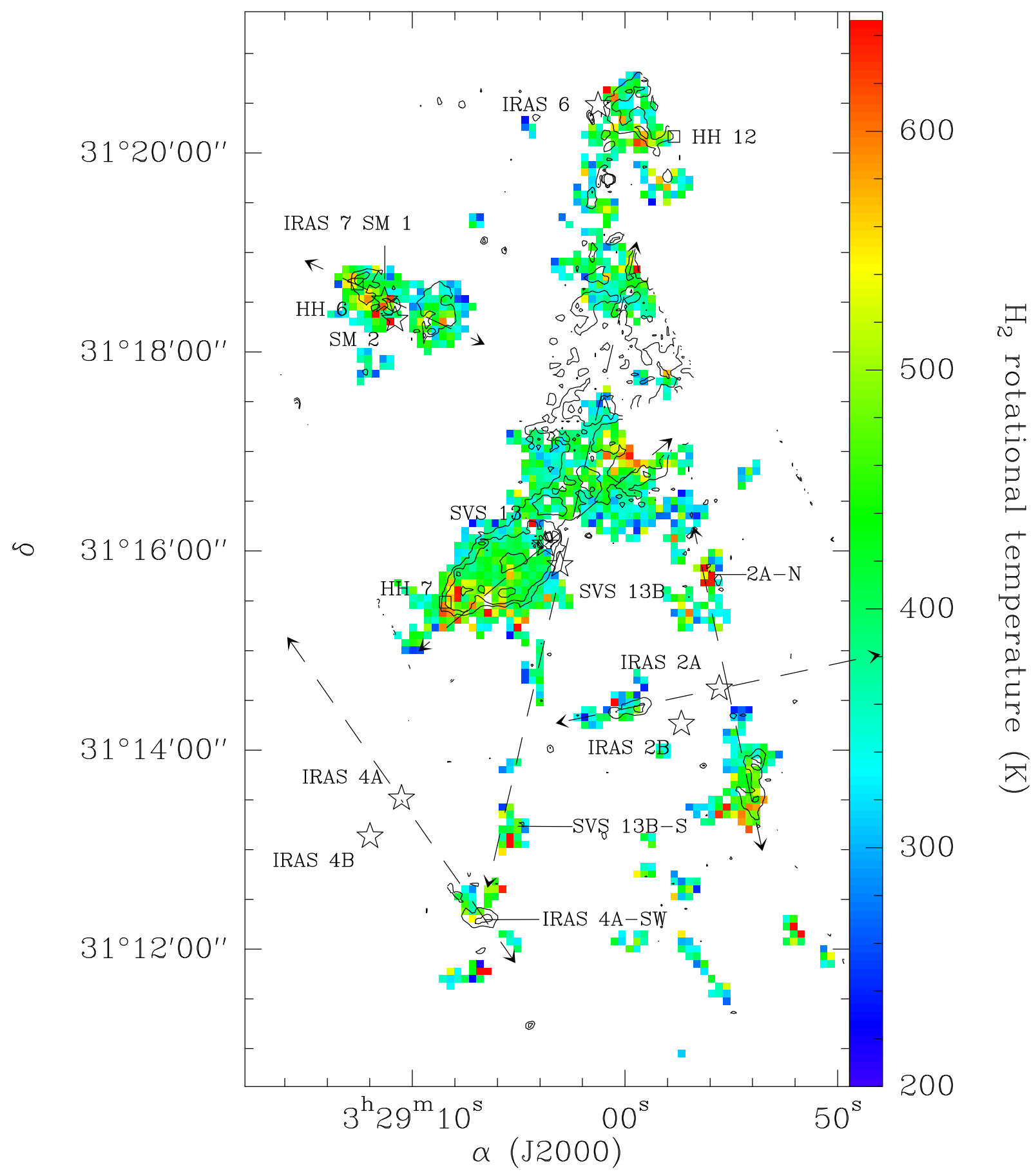

Fig. 7.- Same as Fig. 6 for the $\mathrm{H}_{2}$ rotational temperature. 
NGC 1333

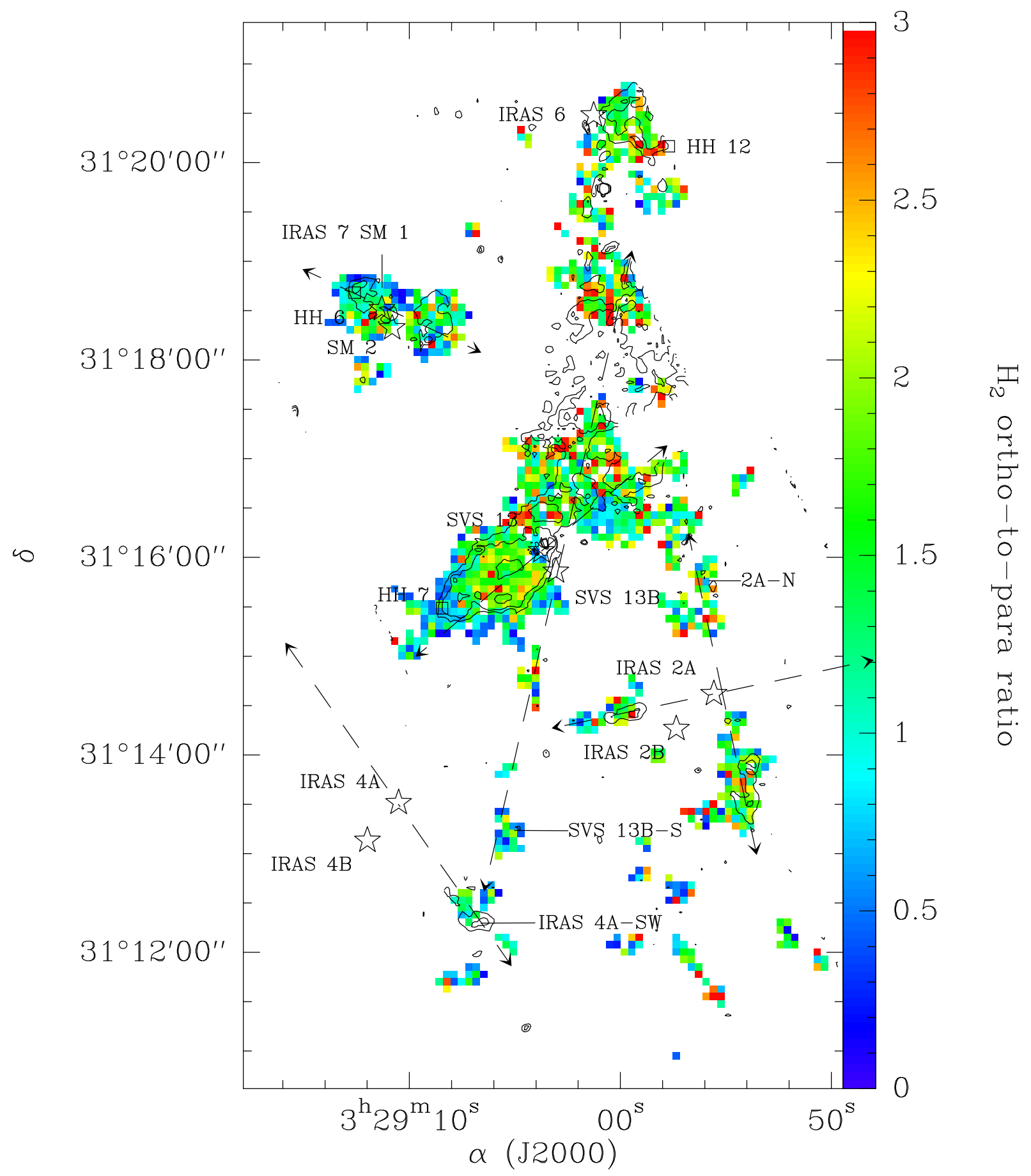

Fig. 8.- Same as Fig. 6 for the $\mathrm{H}_{2}$ ortho-to-para ratio. 


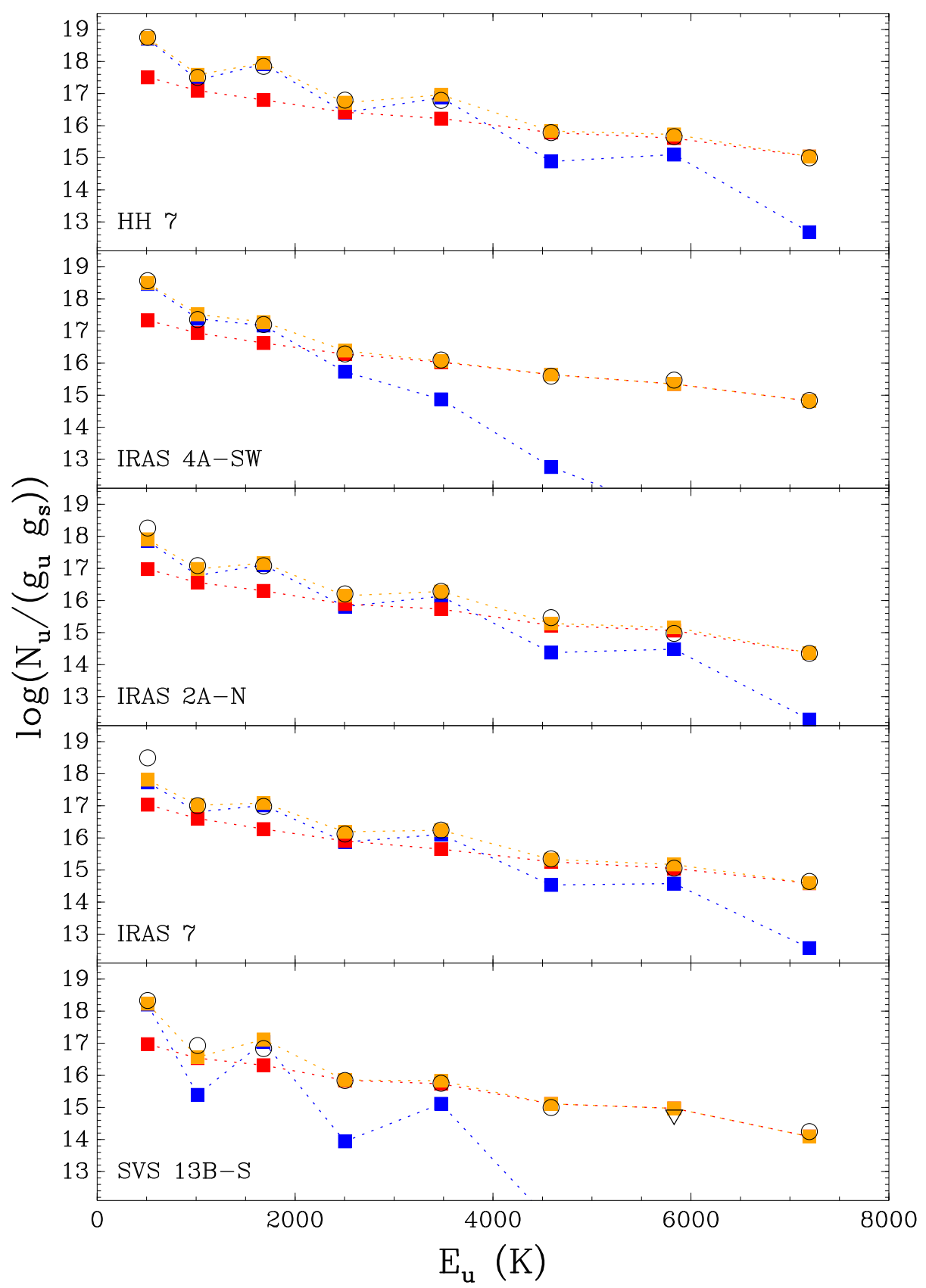

Fig. 9.- Same as in Fig. 5 , but with the two velocity components shock model predictions over plotted (see $\S 5.1$. The blue and red squares connected by dotted lines show the emission from the slow and fast shock components, respectively. The orange squares connected by dotted lines show the total emission from these two components. Note that some of the blue squares (at low energies), as well as some of the red squares (at high energies) are masked by the orange squares. 


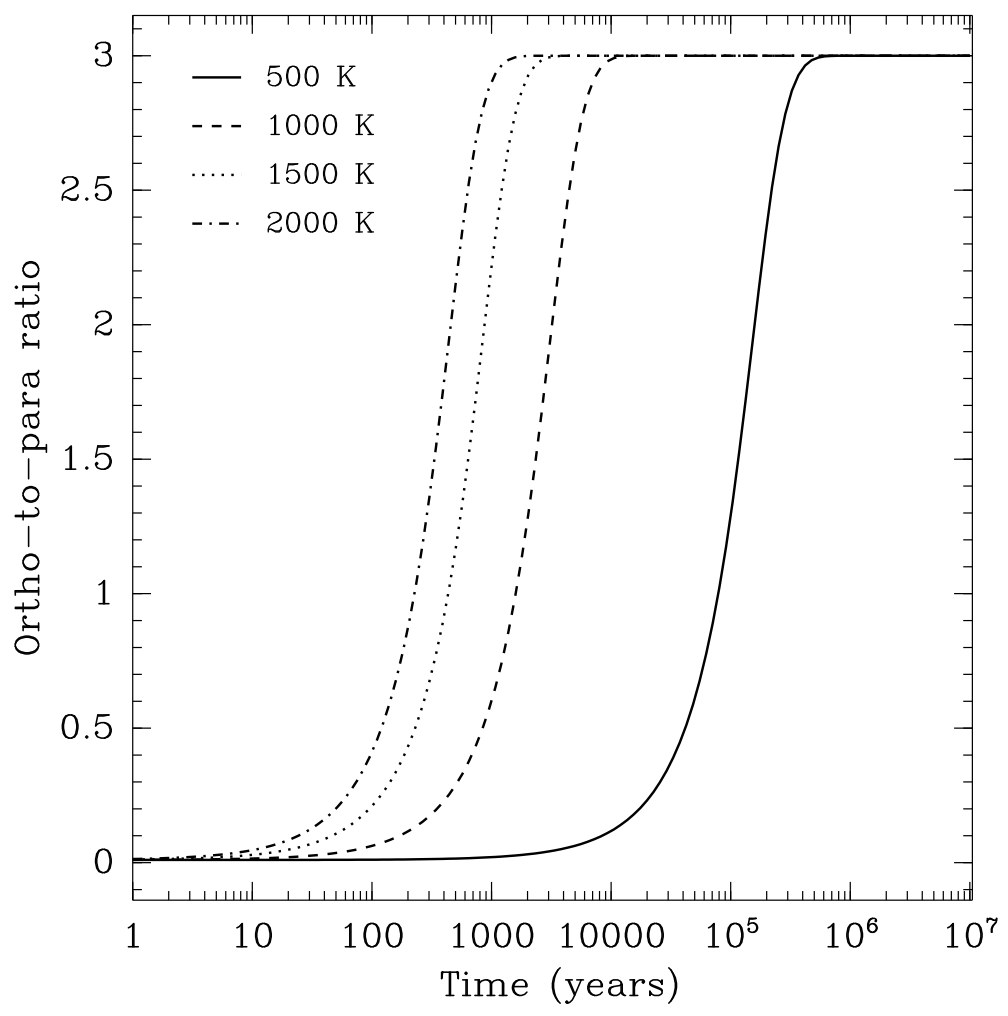

Fig. 10.- $\mathrm{H}_{2}$ ortho-to-para ratio as a function of time for different gas temperatures. An initial opr of 0.01 and $\mathrm{H}$ density of $10 \mathrm{~cm}^{-3}$ are assumed for all curves.

Table 4. Outflow properties

\begin{tabular}{lccccccc}
\hline \hline Source & $\begin{array}{c}\mathrm{L}_{\mathrm{H}_{2}} \\
\left(10^{-2} \mathrm{~L}_{\odot}\right)\end{array}$ & $f_{c}$ & $\begin{array}{c}v_{s} \\
\left(\mathrm{~km} \mathrm{~s}^{-1}\right)\end{array}$ & $\begin{array}{c}\dot{M}_{w} \\
\left(\mathrm{M}_{\odot} \mathrm{yr}^{-1}\right)\end{array}$ & $\begin{array}{c}\dot{P} \\
\left(\mathrm{M}_{\odot} \mathrm{yr}^{-1} \mathrm{~km} \mathrm{~s}^{-1}\right)\end{array}$ & $\begin{array}{c}\tau_{d y n}{ }^{\mathrm{a}} \\
(\mathrm{yr})\end{array}$ & $\begin{array}{c}P \\
\left(\mathrm{M}_{\odot} \mathrm{km} \mathrm{s}^{-1}\right)\end{array}$ \\
\hline HH 7-11 & 11.1 & 0.50 & 26 & $2 \times 10^{-6}$ & $5 \times 10^{-5}$ & 5600 & 0.29 \\
IRAS 4A & 1.8 & 0.25 & 16 & $2 \times 10^{-6}$ & $3 \times 10^{-5}$ & 12000 & 0.38 \\
IRAS 2 $^{\mathrm{b}}$ & 1.5 & 0.25 & 28 & $6 \times 10^{-7}$ & $2 \times 10^{-5}$ & 17000 & 0.34 \\
IRAS 7 & 1.8 & 0.25 & 34 & $5 \times 10^{-7}$ & $2 \times 10^{-5}$ & 5700 & 0.11 \\
SVS 13B & 0.8 & 0.25 & 19 & $7 \times 10^{-7}$ & $2 \times 10^{-5}$ & 22000 & 0.44 \\
\hline
\end{tabular}

${ }^{a}$ From Knee \& Sandell (2000)

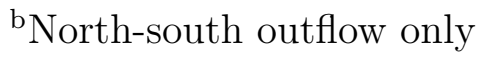




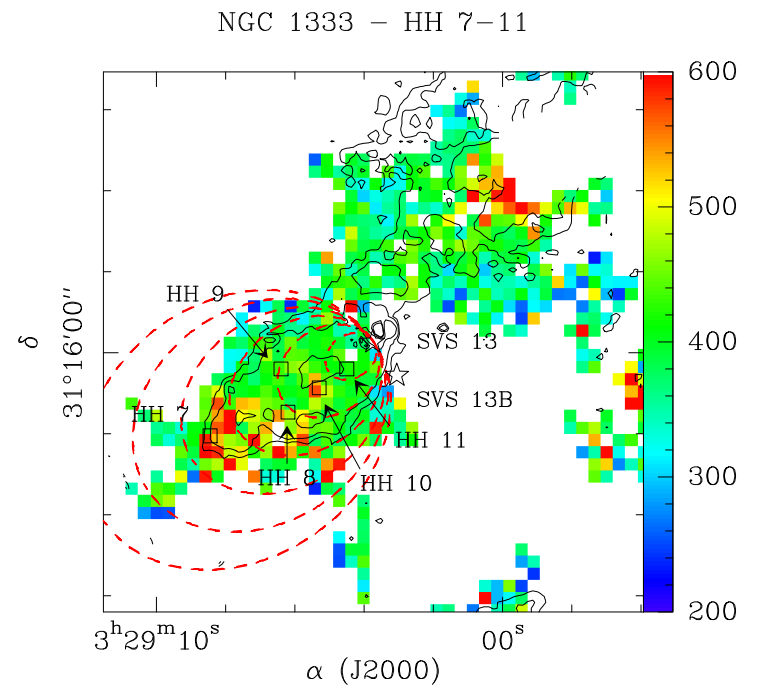

NGC 1333 - IRAS 4

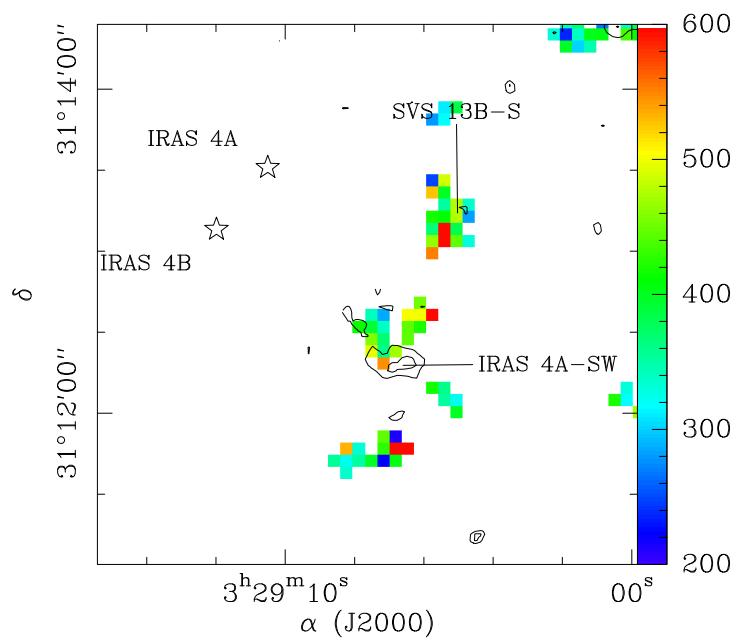

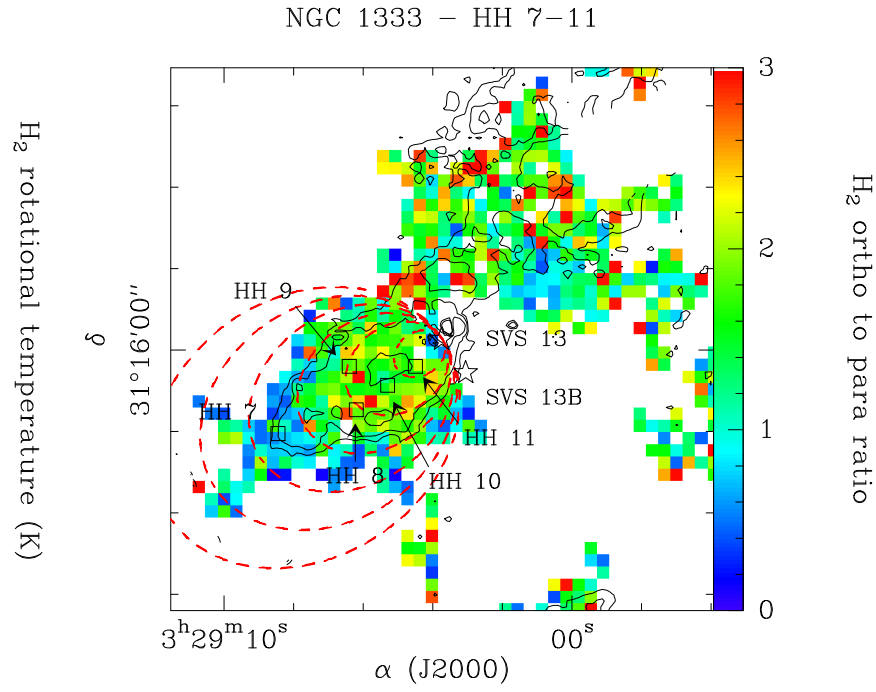

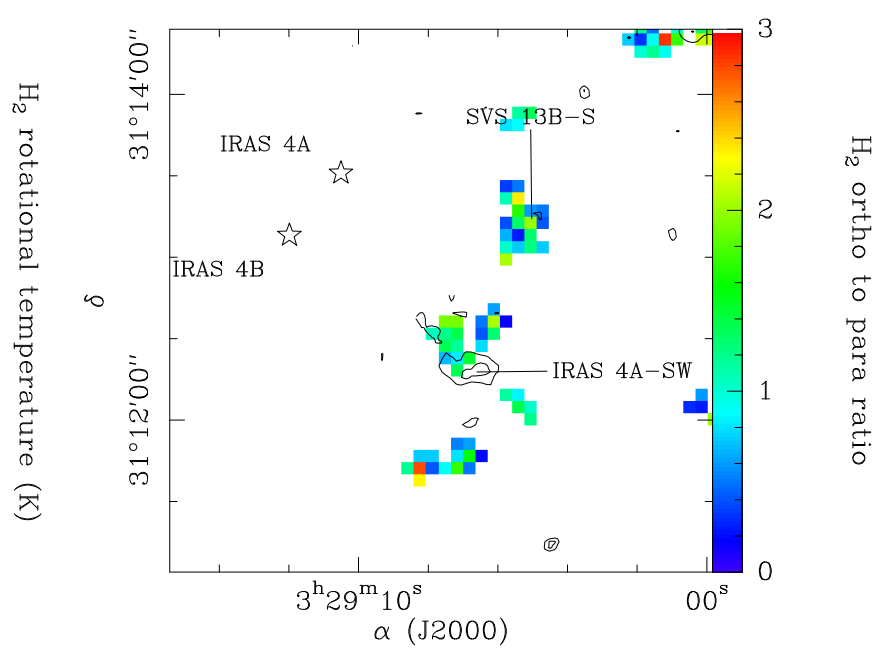

Fig. 11. - Side-by-side comparison between the rotational temperature and opr around SVS 13 (upper panels) and IRAS 4 (lower panels). The position of several HH objects around SVS 13, from Walawender et al. (2005), are indicated. The ellipses that were used to average the opr and the rotational temperature (see $\S \mathrm{A}$ ) are shown. 


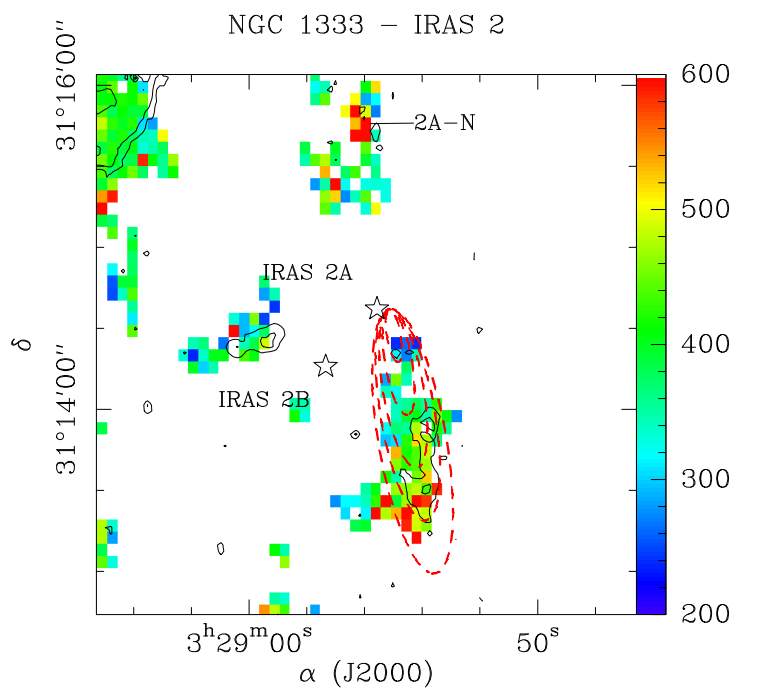

NGC 1333 - IRAS 7

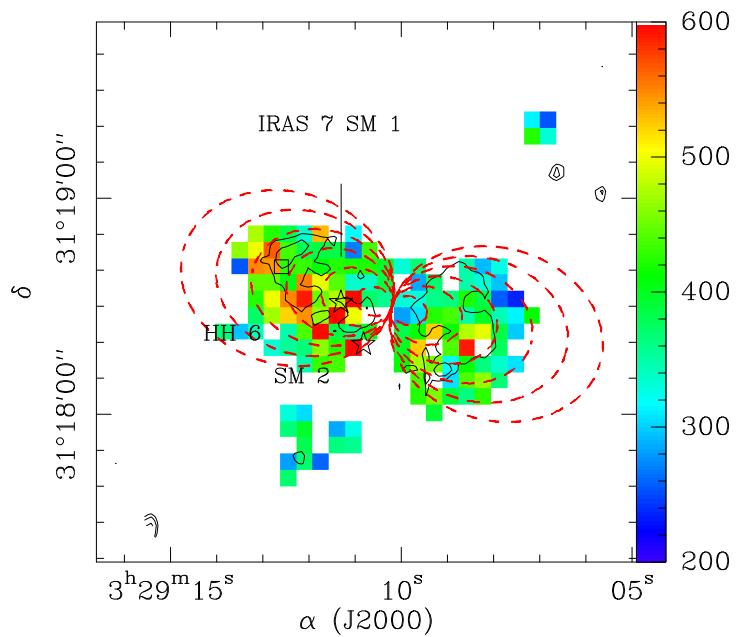

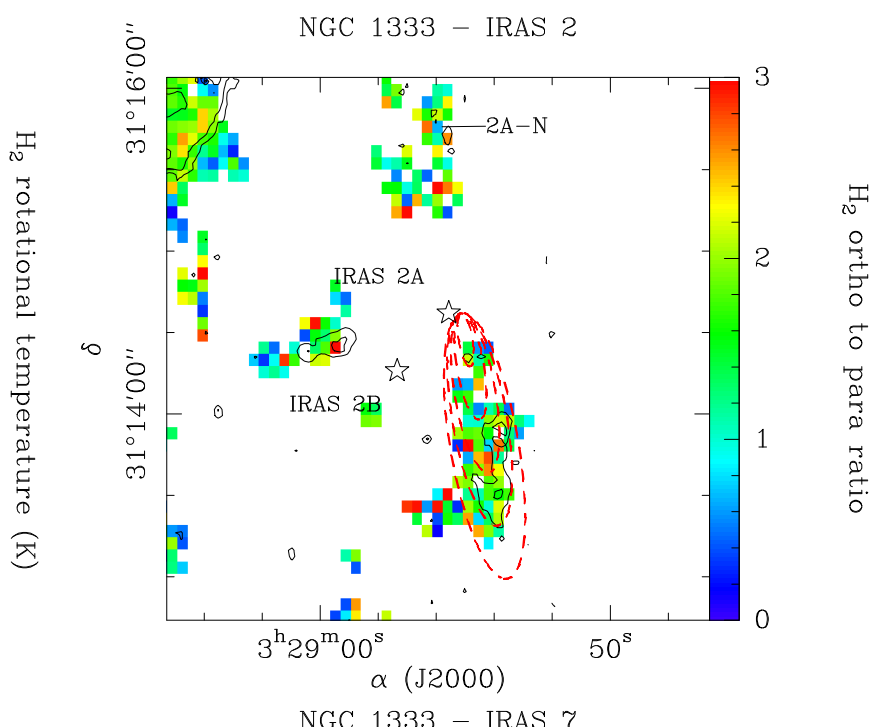

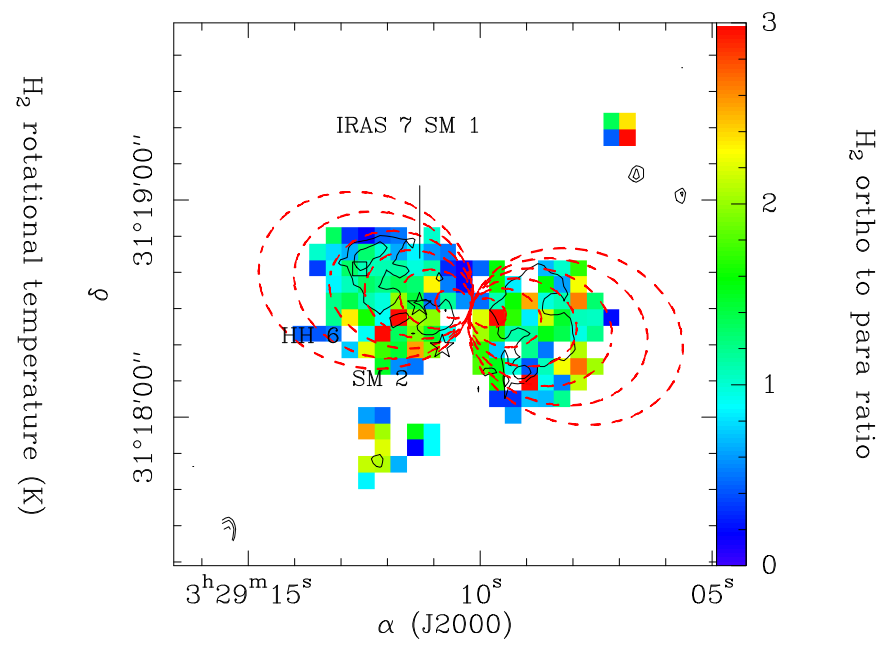

Fig. 12.- Same as Fig. 11 for the IRAS 2 (upper panels) and IRAS 6 (lower panels). 

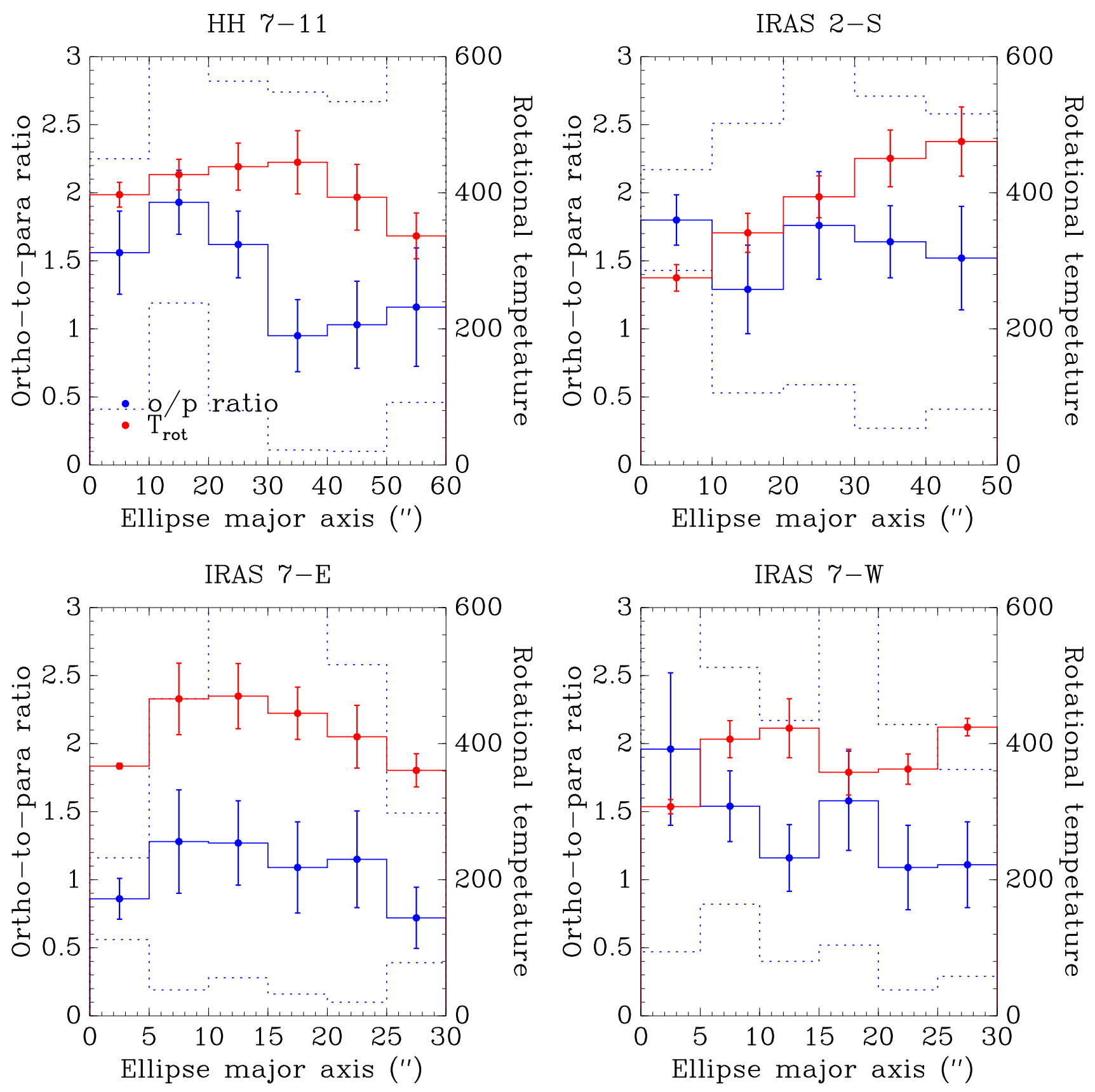

Fig. 13. - Average $\mathrm{H}_{2}$ ortho-to-para ratio (blue histograms in solid lines) and rotational temperature (red histograms) between consecutive ellipses of the same eccentricity, as a function of the ellipse semi-major axis. The error bars are $1 \sigma$ standard deviations computed from the variance in each bin (i.e. the region between two ellipses). The blue histogram in dotted lines show the maximum and minimum values of the opr in each bin. 


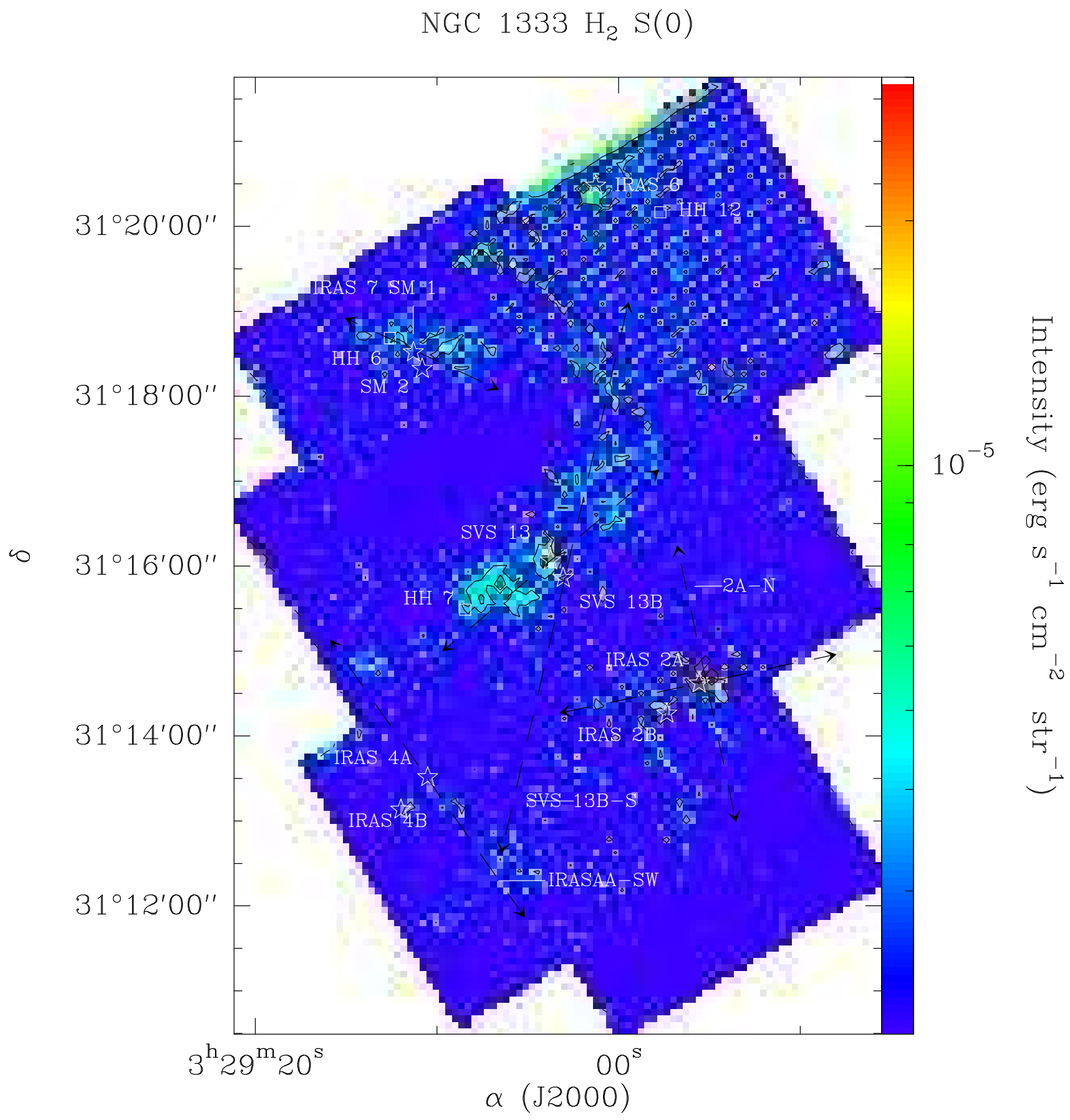

Fig. 4 d. $-\mathrm{H}_{2} S(0)$ map obtained with the LH module. Contour levels are drawn at $3 \sigma$. 


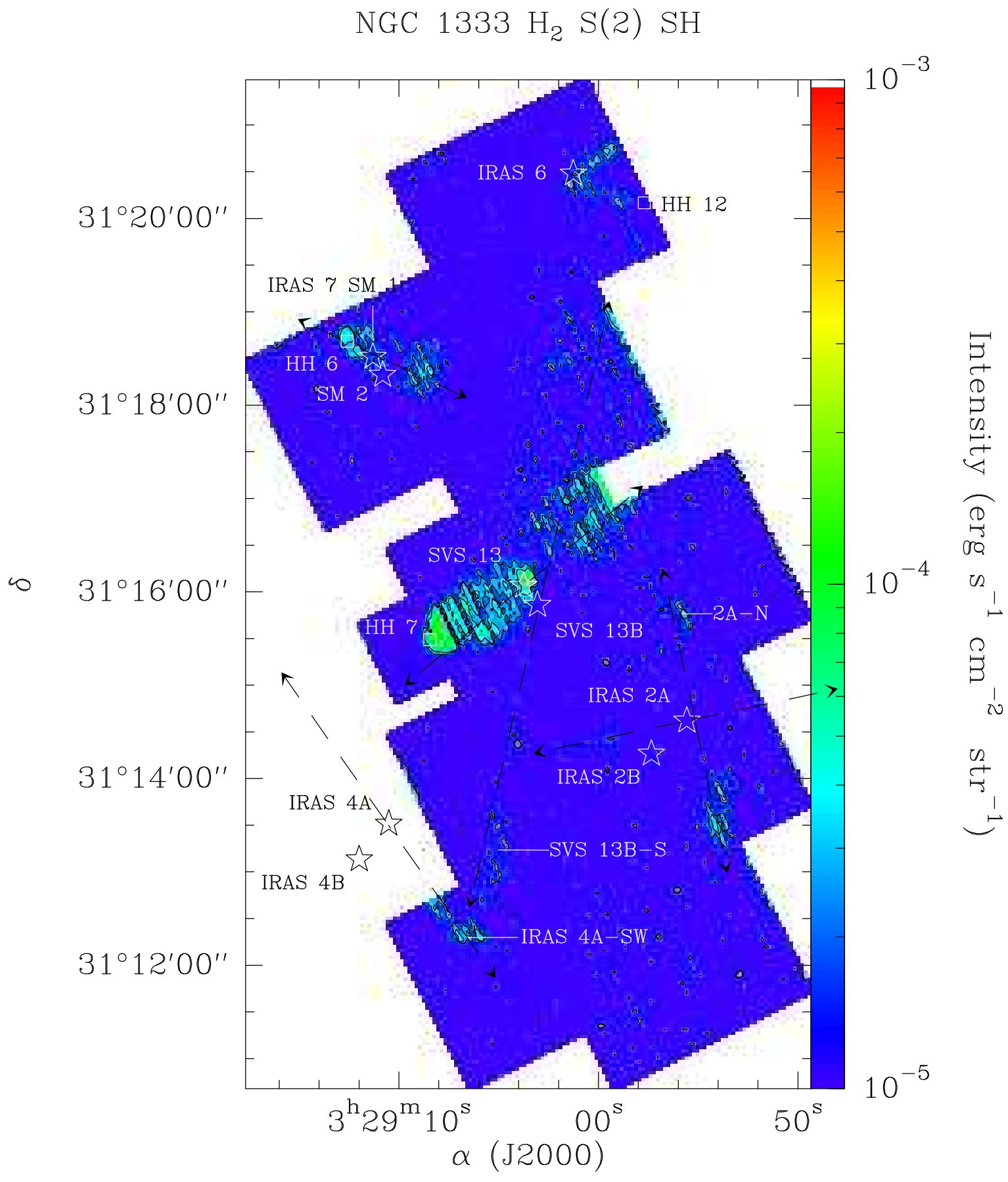

Fig. 4 e. $-\mathrm{H}_{2} S(2)$ map obtained with the $\mathrm{SH}$ module. Contours show the 3 and $5 \sigma$ noise levels. 


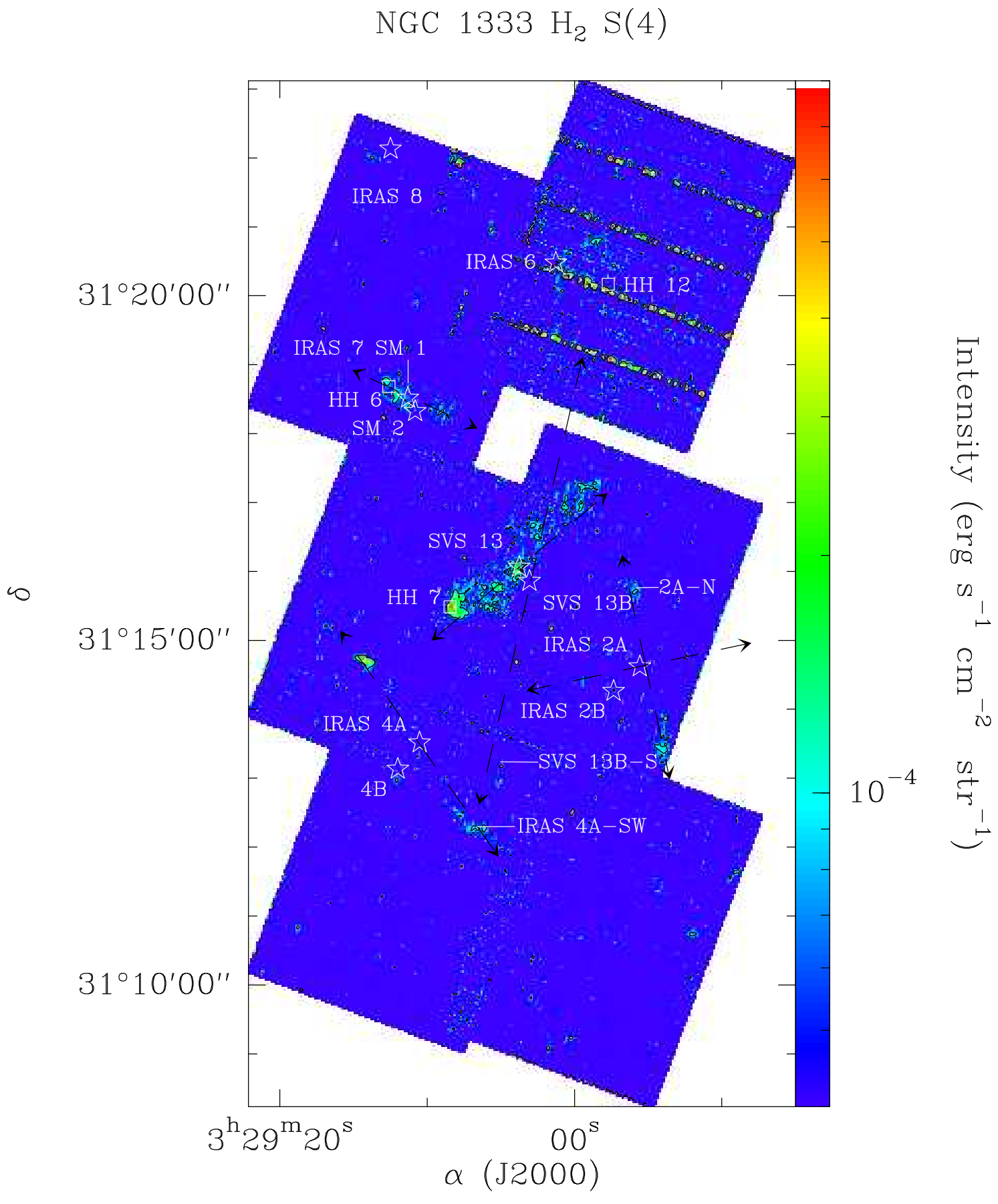

Fig. 4 f. $-\mathrm{H}_{2} S(4)$ map obtained with the SL module. Contours show the $3 \sigma$ noise level. 


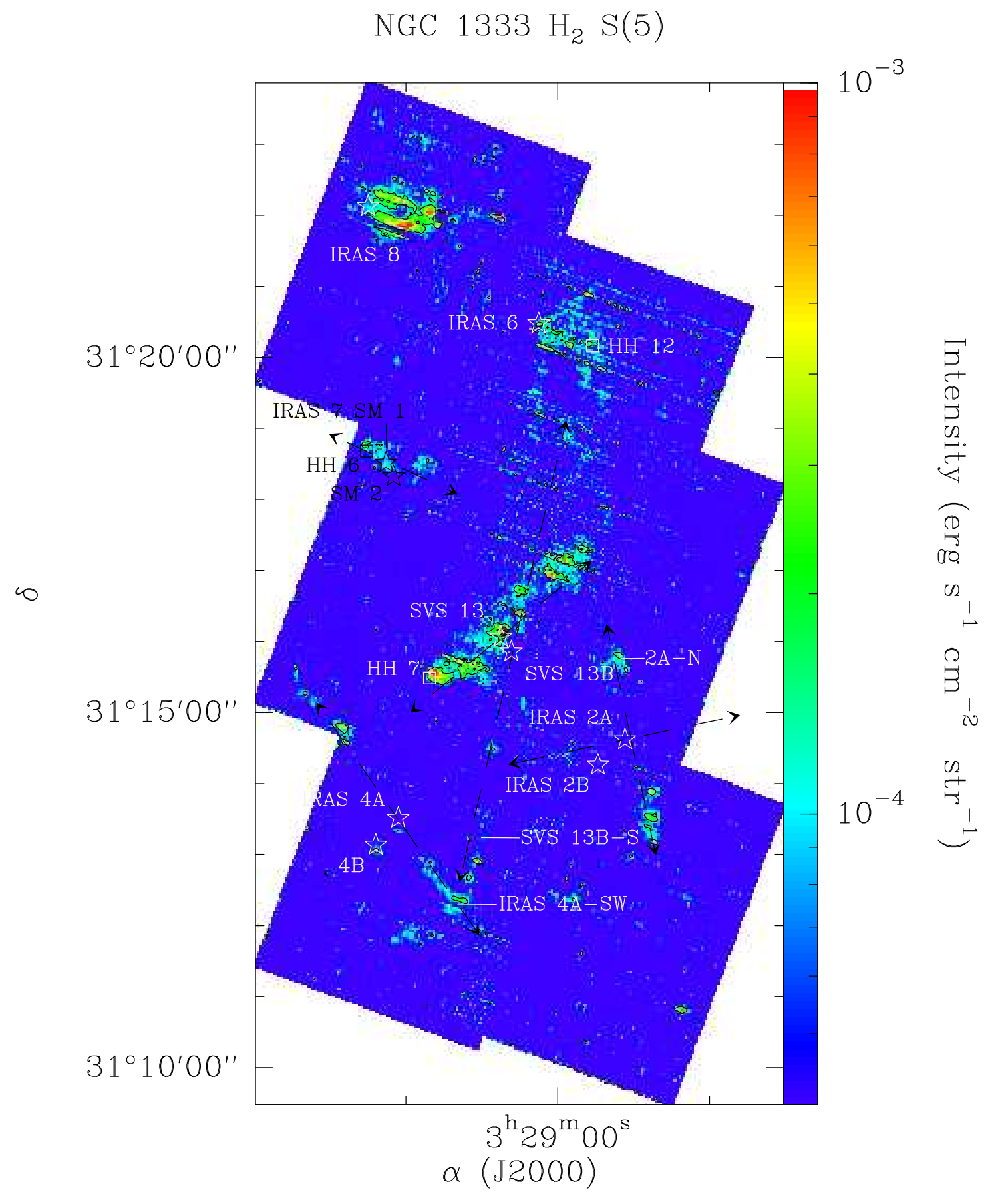

Fig. 4 g. $-\mathrm{H}_{2} S(5)$ map obtained with the SL module. Contours show the $3 \sigma$ noise level. 


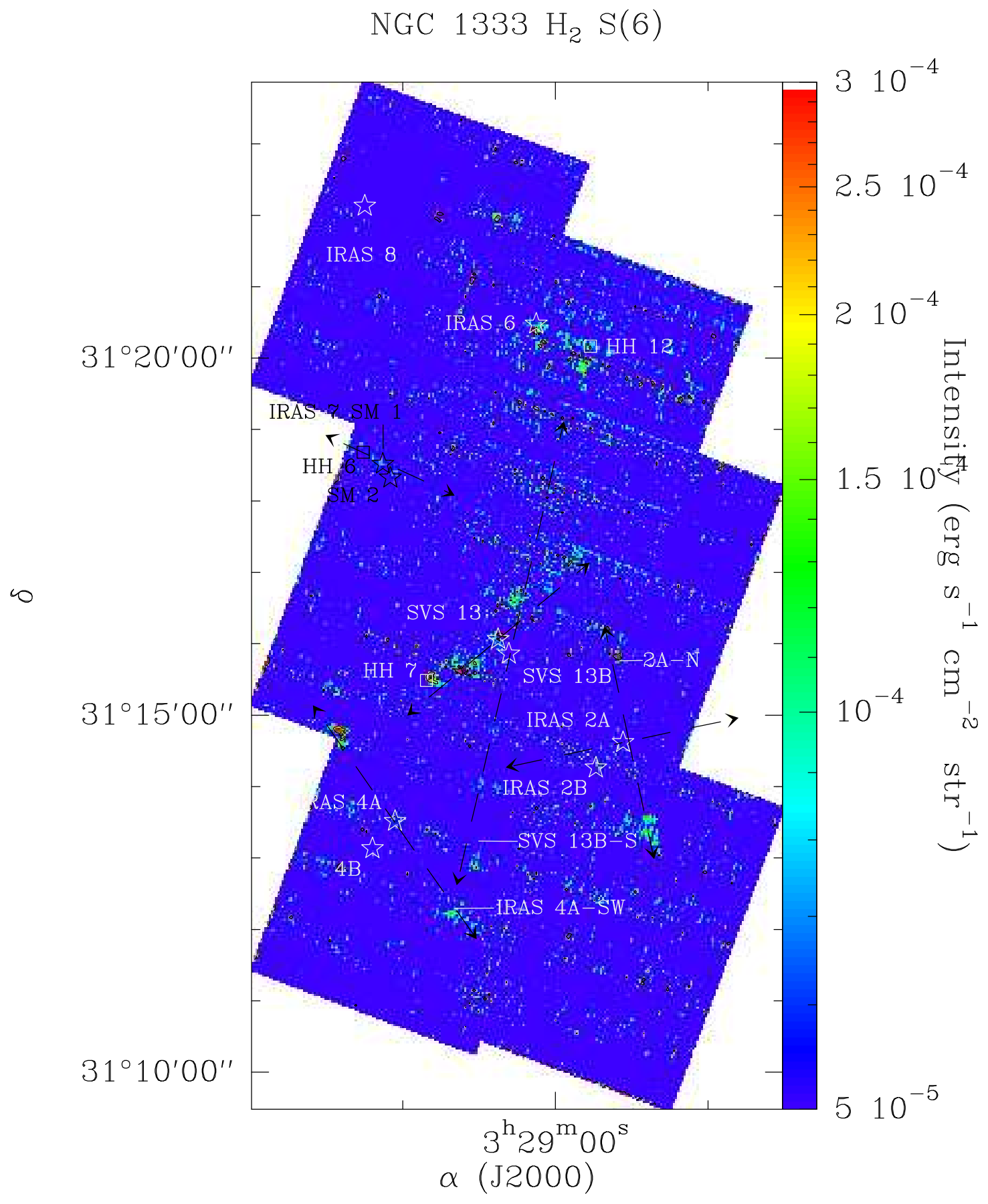

Fig. 4 h. $-\mathrm{H}_{2} S(6)$ map obtained with the SL module. Contours show the $3 \sigma$ noise level. 


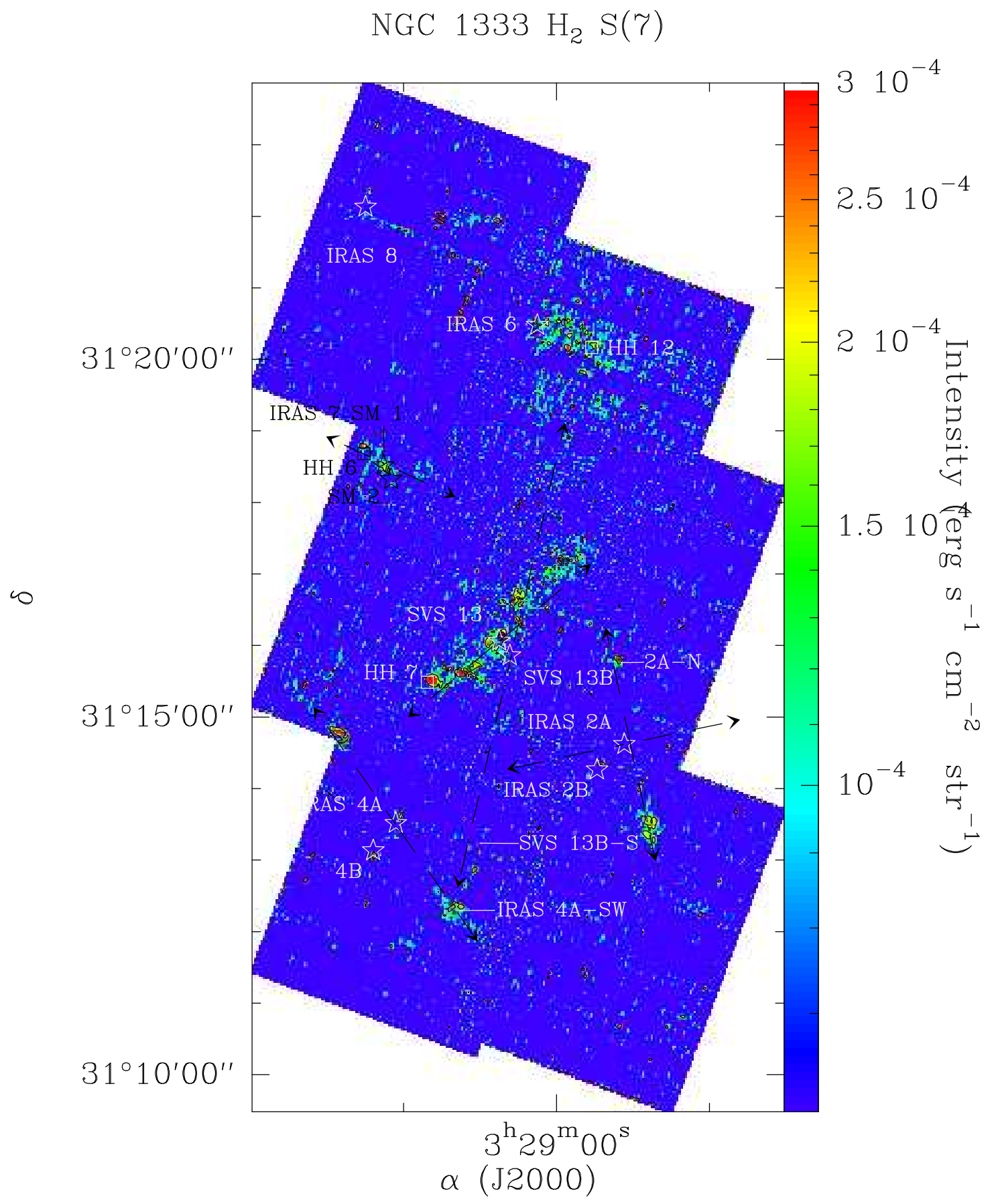

Fig. 4 i. $-\mathrm{H}_{2} S(7)$ map obtained with the SL module. Contours show the $3 \sigma$ noise level. 